\title{
Stabilization of Numerical Interchange in Spectral-Element Magnetohydrodynamics
}

\author{
C. R. Sovinec \\ University of Wisconsin-Madison, Department of Engineering Physics, 1500 Engineering Drive, \\ Madison, Wisconsin 53706-1609
}

\begin{abstract}
Auxiliary numerical projections of the divergence of flow velocity and vorticity parallel to magnetic field are developed and tested for the purpose of suppressing unphysical interchange instability in magnetohydrodynamic simulations. The numerical instability arises with equal-order $C^{0}$ finite- and spectral-element expansions of the flow velocity, magnetic field, and pressure and is sensitive to behavior at the limit of resolution. The auxiliary projections are motivated by physical field-line bending, and coercive responses to the projections are added to the flow-velocity equation. Their incomplete expansions are limited to the highest-order orthogonal polynomial in at least one coordinate of the spectral elements. Cylindrical eigenmode computations show that the projections induce convergence from the stable side with first-order ideal-MHD equations during $h$-refinement and $p$-refinement. Hyperbolic and parabolic projections and responses are compared, together with different methods for avoiding magnetic divergence error. The projections are also shown to be effective in linear and nonlinear time-dependent computations with the NIMROD code [C. R. Sovinec, et al., J. Comput. Phys. 195 (2004) 355-386], provided that the projections introduce numerical dissipation.
\end{abstract}

Keywords: MHD computation, spectral elements, spectral filtering, interchange instability

\section{Introduction}

Magnetohydrodynamic (MHD) computations of magnetically confined plasma must address the numerical realization of localized interchange. In the absence of resistive dissipation, i.e. ideal MHD, the threshold for linear instability lies at a finite amount of "bad" magnetic curvature $[1,2]$. Bending of magnetic field-lines provides a restoring force, but it vanishes for resonant helical perturbations that align wavefronts with magnetic field $(\mathbf{B})$ over entire surfaces of closed field-lines. Mathematically, the model is singular at these surfaces, and numerical representation of the resonant effect influences convergence properties [3-6]. The challenge lies in the fact that responses depend on numerical behavior at the limit of spatial resolution, where convergent numerical methods usually have their greatest level of truncation error. Convergence on local interchange "from the unstable side" or "from above" means having too large a growth rate at finite resolution of a physical instability or producing numerical interchange for physically stable conditions. Lütjens and Luciani aptly recognized this as a problematic source of numerical noise in nonlinear simulations of long timescale dynamics [6]. This article describes a practical approach to addressing numerical interchange in simulations that use finite- and spectral-element representations of $C^{0}$ continuity across element borders. It is conceptually related to spectral 
filtering of incompressible fluid simulations [7], to a penalty method for MHD eigenvalue computations [4], and to finite/spectral element stabilization techniques [for example, 8-11].

Our interest is nonlinear time-dependent simulation of nonideal MHD for magnetic confinement. This model admits viscosity that can stabilize localized interchange and facilitate computations. However, dissipation of dynamics perpendicular to magnetic field is weak in high-temperature plasma, and adding enough conventional viscosity to stabilize numerical interchange can distort dynamics. In principle, it is possible to address numerical interchange with sufficiently fine meshing for physical levels of viscosity. However, resonances that are susceptible to interchange can exist across the entire region of closed magnetic flux, and computing with globally fine resolution is computationally challenging and inefficient. The many resonances that occur in a region of bad magnetic curvature would also foil attempts to stabilize selected modes with spatially localized viscous dissipation.

The numerical representation of interchange was first studied in the context of ideal-MHD eigenvalue computation for static equilibria. The fundamental dependent fields are the components of the displacement vector, and perturbations in pressure and magnetic field are eliminated analytically prior to discretization. The resulting force operator is a second-order differential operator in space, and it is self-adjoint [12]. Finite-element methods (FEM) for this problem benefit from distinct expansions for the flux-normal, parallel, and "cross" components of the displacement vector, and integration by parts leaves only first-order derivatives of basis and test functions. To conform with analytical variational methods, only the radial component in cylindrical computations needs $C^{0}$ continuity in the radial direction $[3,13]$. The other components may be discontinuous, and the degree of polynomials may be chosen to represent incompressible displacements without truncation error within elements. This allows the cylindrical computations to distinguish shear, parallel compression, and perpendicular compression at the scale of the mesh, thereby avoiding numerical spectral pollution. Like other applications of FEM, the conforming representation for low-order elements tends to be too stiff, and weakly growing internal modes in shaped cross sections are easily missed with slow convergence from the stable side [14]. Hybrid methods include auxiliary expansions for element-averaged dependent fields to improve the representation of bending and to achieve convergence from the unstable side [3]. Degtyarev and Medvedev analyzed the hybrid methods and refined them by introducing a numerical penalty term with a resolution-dependent coefficient that enhances bending-like energy at the finest scale of a mesh [4].

The early work on ideal-MHD eigenvalue computation provides a basis for time-dependent computation with weak dissipation, but the latter requires separate expansions for flow velocity, magnetic field, pressure (or temperature), and particle or mass density. When solving equations for the primitive physical fields, ideal effects, including the stabilizing bending response, are represented through first-order spatial derivatives in temporally first-order equations. Although the equation for the displacement vector in eigenvalue computation and the ideal part of timedependent equations represent the same physical system, they are mathematically distinct and require different numerical approaches. The one taken by Lütjens and Luciani expands magnetic flux and the streamfunction for flow with polynomials of different degree [6]. Results described in Sect. 4 concur with their finding that this method achieves convergence on interchange from 
the stable side. However, it allows other numerical modes when using primitive-field expansions in our spectral-element computations.

The approach presented here takes advantage of the spectral-element representation, which allows bases of arbitrary polynomial degree $[15,16]$. We project flow divergence and parallel vorticity onto incomplete, discontinuous polynomial expansions that include only the highestorder orthogonal polynomial in at least one of the element coordinates. Mathematically coercive responses to these projections are added to the evolution equations for components of flow velocity. This improves the numerical stability of the MHD computations, similar to other stabilization methods for FEM. Like spectral filtering [7], the auxiliary responses act on the shortest scales of the representation. In practice their effect on resolved scales is negligible.

The following section describes the equations and local interchange. Section 3 presents the new projection-based approach for stabilizing numerical interchange. Section 4 shows results from numerical eigenvalue computations for numerical systems with and without the new projections. Linear and nonlinear time-dependent results are presented in Sect. 5, followed by discussion and conclusions in Sect. 6.

\section{Equations and local interchange}

The nonlinear non-ideal MHD system is the model of interest for applications to magnetized plasma, but their linearized form in the ideal limit is useful for investigating numerical interchange behavior. Thus, both are presented in the first part of this section. The second part describes the gravitational instability in a sheared-slab configuration as a relatively simple realization of MHD interchange. It highlights the importance of responses to parallel vorticity and compression, motivating the numerical approaches that are introduced in Sect. 3.

\subsection{Nonlinear and linear MHD equations}

The developments described in this paper have been applied to the NIMROD (Non-Ideal MHD with Rotation) code [17], which solves time-dependent nonlinear and linear problems of extended MHD. In normalized units, the most basic nonlinear single-fluid system solved by NIMROD is

$$
\begin{aligned}
& \left(\frac{\partial}{\partial t}+\mathbf{V} \cdot \nabla\right) \mathbf{V}=\mathbf{J} \times \mathbf{B} \quad \nabla P \quad \nabla \cdot{ }_{-}, \\
& \frac{\partial}{\partial t}+\nabla \cdot(\mathbf{V})=\nabla \cdot\left(\begin{array}{ll}
D_{n} \nabla & D_{h} \nabla \nabla^{2}
\end{array}\right), \\
& \frac{1}{1} \frac{m}{m}\left(\frac{\partial}{\partial t}+\mathbf{V} \cdot \nabla\right) T=\frac{P}{2} \nabla \cdot \mathbf{V} \quad \nabla \cdot \mathbf{q}, \\
& \frac{\partial \mathbf{B}}{\partial t}=\nabla \times \mathbf{E}+\nabla, \\
& \mathbf{J}=\nabla \times \mathbf{B},
\end{aligned}
$$


where $\rho$ and _ in the flow-velocity equation (1) are the mass density and a viscous stress tensor, respectively. The equation for temperature (3) includes a conductive heat-flux density $\mathbf{q}$, and $\Gamma$ is the adiabatic index, which is typically set to $5 / 3$. When modeling electron and singly charged ion species assuming rapid thermal equilibration, the plasma pressure is $P=2 T / m$, where $m$ is the ion mass. The diffusive and hyper-diffusive terms on the right side of the continuity equation (2) are included for numerical smoothing. In ideal MHD, the electric field E in Faraday's law (4) is simply $\mathbf{E}=\mathbf{V}$ B. Resistive MHD includes $\mathbf{J}$ with $\eta$ being the electrical resistivity.

The last term on the right side of Eq. (4) is numerical. It is used to enforce the magnetic divergence constraint, $\nabla \cdot \mathbf{B}=0$, that is part of the physical model. The spectral-element representation in NIMROD is not able to satisfy a divergence constraint exactly, but errors can be controlled quite well [17]. Diffusive error control with $={ }_{b} \nabla \cdot \mathbf{B}$ is the standard approach for NIMROD computations. However, as shown in Sect. 4, the method of controlling magnetic divergence errors influences the behavior of numerical interchange. Thus, different forms of $\Phi$ are reviewed in Sect. 3 and are compared in Sect. 4 when applying the new projections for flow divergence and parallel vorticity.

The most important sources of free energy for magnetic confinement are represented in the ideal version of system (1-5) for small perturbations about stationary equilibria $\left(\mathbf{J}_{0} \times \mathbf{B}_{0}=\nabla P_{0}\right.$; $\left.\mathbf{V}_{0}=\mathbf{0}\right)$. The equations are linear in the dependent variables for perturbed flow velocity $(\mathbf{v})$, mass density $(\rho)$, pressure $(p)$, and magnetic field $(\mathbf{b})$ :

$$
\begin{aligned}
& 0 \frac{\partial}{\partial t} \mathbf{v}=(\nabla \times \mathbf{b}) \times \mathbf{B}_{0}+\mathbf{J}_{0} \times \mathbf{b} \nabla p \\
& \frac{\partial}{\partial t}+\mathbf{v} \cdot \nabla_{0}=\quad{ }_{0} \nabla \cdot \mathbf{v}, \\
& \frac{\partial}{\partial t} p+\mathbf{v} \cdot \nabla P_{0}=\quad P_{0} \nabla \cdot \mathbf{v}, \\
& \frac{\partial}{\partial t} \mathbf{b}=\nabla \times\left(\mathbf{v} \times \mathbf{B}_{0}\right)+\nabla .
\end{aligned}
$$

They may also be expressed in terms of the mechanical displacement vector $\boldsymbol{\xi}$, for $\mathbf{V}_{0}=\mathbf{0}$ we may substitute $\partial \xi / \partial t=\mathbf{v}$ and integrate (7-9) in time. Assuming solutions of the form $\exp (i t)$ and dropping the numerical $\nabla$ term in Eq. (9) leads to the standard ideal-MHD eigenvalue equation [12],

$$
-\rho_{0} \omega^{2} \boldsymbol{\xi}=\left[\nabla \times \nabla \times\left(\xi \times \mathbf{B}_{0}\right)\right] \times \mathbf{B}_{0}+\mathbf{J}_{0} \times \nabla \times\left(\xi \times \mathbf{B}_{0}\right)+\nabla\left(\xi \cdot \nabla P_{0}+\Gamma P_{0} \nabla \cdot \xi\right),
$$

where the right side is the self-adjoint linear force operator $\mathbf{F}(\xi)$. When modeling a closefitting wall, boundary conditions set the normal component of displacement along the wall to zero.

\subsection{Gravity-driven interchange}


A relatively simple analog for pressure-driven interchange inserts the gravitational force density $\mathbf{g}$ in Eq. (6) or Eq. (10) and considers slab geometry, where complications from magnetic curvature are removed. Having equilibrium density increasing with height $y$ allows Rayleigh-Taylor like instability that is mathematically similar to pressure-driven interchange $[12,18]$ for wavelengths that are short relative to the scale of equilibrium variation $L$.

Considering $\mathbf{g}=g \hat{\mathbf{e}}_{y}$ in equilibria with $B_{0_{y}}=0$ and uniform $P_{0}$ and $B_{0_{z}}$ implies

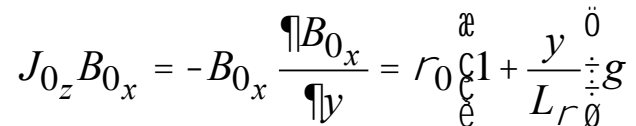

near $y=0$, where $\quad 0$ is now taken as a constant. Perturbed fields of the form $f(y) \exp \left[\begin{array}{lll}i k_{x} x+i k_{z} z & i & t\end{array}\right]$ have wavefronts that align with $\mathbf{B}_{0}$ at $y=0$ when $\mathbf{k}$ is chosen to satisfy the resonance condition $\mathbf{k} \times\left.\mathbf{B}_{0}\right|_{y=0}=0$. Defining $F \quad \mathbf{k} \times \mathbf{B}_{0} \quad F y$, where the prime indicates $/ y$, the system simplifies to

$$
\left(F^{2}-\rho_{0} \omega^{2}\right) \xi=-\nabla\left[i F \xi \cdot \mathbf{B}_{0}+J_{0_{z}} B_{0_{x}} \xi_{y}-\left(B_{0}^{2}+\Gamma P_{0}\right) \nabla \cdot \xi\right]-i F \mathbf{B}_{0} \nabla \cdot \xi+\frac{\rho_{0} g}{L_{\rho}} \xi_{y} \hat{\mathbf{e}}_{y} .
$$

The above expression includes the compression of fluid and magnetic pressures, but it drops the compressive gravitational term, $g \rho_{0} \nabla \cdot \xi$, which is not part of the analogy for pressure-driven interchange.

When combined, the components of displacement along $\mathbf{k}$ and along $\mathbf{B}_{0}$ imply that the divergence of displacement satisfies

$$
\left(k^{2} S-\rho_{0}^{2} \omega^{4}\right) \nabla \cdot \xi=A\left(k^{2} J_{z} B_{x} \xi_{y}-\rho_{0} \omega^{2} \xi_{y}^{\prime}\right),
$$

where

$$
\begin{array}{lcc}
A & 0^{2} F^{2}, \text { and } \\
S & 0 & 2\left(B_{0}^{2}+P_{0}\right) \quad F^{2} P_{0} .
\end{array}
$$

Eliminating the divergence of flow from the $y$-component of (11) leads to the scalar eigenvalue equation

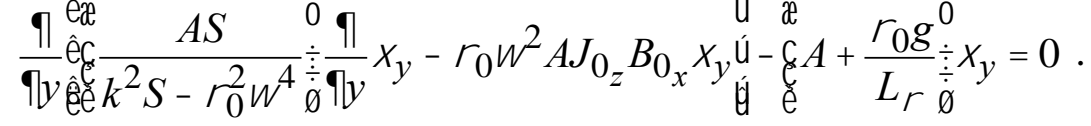

For low frequency dynamics, including all unstable modes in realistic configurations, $k^{2} S \gg{ }_{0}^{2}{ }^{4}$, so the factor in the highest-order term is approximately $A k^{2}$. It includes the $F^{2} k^{2} \quad F^{2} y^{2} k^{2}$ term from field-line bending that makes Eq. (13) singular in the limit of vanishing 2 . 
Near marginal stability, the nonsingular terms with factors of $A$ are negligible, and the relation simplifies to

$$
-\left(\begin{array}{lll}
0^{2} F^{2} & y^{2}
\end{array}\right)-D_{s} y=0
$$

which can also be obtained using incompressibility, $\nabla \cdot \xi=0$, in place of Eq. (8). The factor $D_{s} \quad k^{2}{ }_{0} g / F^{2} L$ is the effective Suydam parameter for this $g$-mode and represents the strength of the gravitational free energy relative to the bending response. The singular bending term $\left(y^{2} y\right)$ has a maximum eigenvalue of 1/4 [6], which compensates free energy and leads to the analytical stability criterion $D_{S}$ 1/4 [1]. Bounded eigenfunctions at larger values of $D_{S}$

can have $2<0$, and those modes grow in time. The unstable modes have significant vorticity parallel to $\mathbf{B}_{0}$ in the vicinity of the resonance, which interchanges magnetic flux tubes to reduce the free energy of the configuration. Because $A \quad 0$ near $y=0$ for conditions of weak instability, Eq. (12) implies that the interchange dynamics described by the more complete Eq. (13) are also essentially incompressible.

The above discussion has implications for numerical solution of systems (1-5) or (6-9), which is labeled the "v, b, $p$-system" in the following sections. First, when solving equations that allow compressibility, the stabilizing influence of field-line bending in Eq. (13) requires an accurate response to compression. With localization arising from the singularity, the numerical representation of compression at the scale of the mesh is important. Second, when solving a system with separate equations for magnetic field and flow velocity (or displacement), the stabilizing field-line bending response is represented in first-order spatial derivatives in separate equations. In a conventional element-based expansion, these terms are not mathematically coercive and may effectively vanish at the mesh scale. The first issue also arises in eigenvalue computations with the second-order form of Eq. (10). It has been addressed by using basis functions of differing continuity for magnetic-flux components of displacement that are defined with respect to the equilibrium $\mathbf{B}_{0}$ [13]. However, this approach is not suitable for nonlinear time-dependent computation, where $\mathbf{B}$ evolves appreciably. The discontinuous expansions would also require special treatment of physical dissipation. The second issue of losing meshscale bending in first-order systems is related to numerical destabilization with hybrid finite elements $[3,4]$.

\section{Auxiliary responses and formulation}

The proposed approach for stabilizing numerical interchange modifies the MHD equations with auxiliary responses at the limit of a spectral-element representation. The modified equations for flow velocity are described in the first of the following subsections, and their weak forms and function spaces are presented in the second subsection. The alternative approaches for approximating the magnetic divergence constraint that are considered in this investigation of numerical interchange are reviewed in the third subsection.

\subsection{Auxiliary flow-divergence and parallel-vorticity responses}


In the compressible MHD model, the divergence of flow-velocity or displacement induces changes in the plasma-fluid pressure $P$. If the divergence results from motions that are perpendicular to the magnetic field-lines, it also induces changes in the magnetic pressure $B^{2} / 2$. Similarly, if wavefronts are not aligned with $\mathbf{B}$, vorticity parallel to $\mathbf{B}$ bends magnetic field-lines. At the resolved scales of a numerical computation of the compressible MHD equations, these effects excite restoring responses. When using $C^{0}$ elements in a weak form of the first-order $\mathbf{v}$, b, $p$-system, however, conventional Galerkin projection misses some of the nontrivial realizations of divergence and curl at the nodal scale. These considerations motivate adding specialized auxiliary responses to avoid unphysical numerical interchange at the nodal scale.

The hyperbolic form of the auxiliary responses can be viewed as augmenting the force densities from the gradient of pressure (fluid plus magnetic) and from the bending of magnetic field-lines. In differential form, the augmented linear flow-velocity equation and specialized responses are

$$
\begin{aligned}
0 \frac{\partial}{\partial t} \mathbf{v}=(\nabla \times \mathbf{b}) \times \mathbf{B}_{0}+\mathbf{J}_{0} \times \mathbf{b} \quad \nabla p+\nabla\left(f_{d} \sqrt{P_{c}}\right) \nabla \times\left(f_{v} \mathbf{B}_{0}\right) \\
\frac{\partial}{\partial t} \quad=f_{d} \sqrt{P_{c}} \nabla \cdot \mathbf{v} \\
\frac{\partial}{\partial t}=f_{v} \mathbf{B}_{0} \cdot \nabla \times \mathbf{v}
\end{aligned}
$$

where $P_{c} \quad B_{0}^{2}+P_{0}$ is the square of the magneto-acoustic compressional wave speed, $c_{m}^{2}$, multiplied by 0 . The above set of equations replaces Eq. (6) in the system (6-9). The constants $f_{d}$ and $f_{v}$ are dimensionless numerical parameters that affect the rates at which the nodal-scale flow divergence and parallel vorticity are propagated. The auxiliary responses are related to the hyperbolic method of applying constraints that has been used for satisfying charge conservation in Maxwell's equations [19]. However, the compressible MHD model already has physical responses to flow divergence and parallel vorticity. Thus, the auxiliary constraints here must adjust to affect only the finest scale of the computation, where representation of the physical responses is poor.

Parabolic numerical responses provide an alternative to the hyperbolic method described above. The relevant differential form of the augmented linear flow-velocity equation and specialized responses is

$$
\begin{aligned}
& 0 \frac{\partial}{\partial t} \mathbf{v}=(\nabla \times \mathbf{b}) \times \mathbf{B}_{0}+\mathbf{J}_{0} \times \mathbf{b} \quad \nabla p+\nabla\left(\sqrt{d_{d} t P_{c}}\right) \nabla \times\left(\sqrt{d_{v} t} \mathbf{B}_{0}\right) \\
& =\sqrt{d_{d} t P_{c}} \nabla \cdot \mathbf{v} \\
& =\sqrt{d_{v} t} \mathbf{B}_{0} \cdot \nabla \times \mathbf{v}
\end{aligned}
$$

where the time-step $t$ of a temporal advance is inserted to make $d_{d}$ and $d_{v}$ normalized viscosity parameters for flow divergence and parallel vorticity. This parabolic method is related to 
imposing incompressibility in finite-element fluid computations using the penalty method [20], but again the auxiliary constraints here apply to the nodal scales, only.

\subsection{Element-based formulation}

Like other science and engineering applications of element-based methods, solving the nonlinear non-ideal MHD system with equal-order $C^{0}$ bases for the primitive physical fields is convenient. In the NIMROD code, 2D $C^{0}$ elements are combined with finite Fourier series for the third, periodic coordinate. As shown in Ref. [17], allowing polynomial expansions of arbitrary degree is effective for reproducing anisotropic thermal conduction without mesh alignment. It is also effective for approximating the magnetic divergence constraint. The 2D elements use Lagrange-type basis functions for the physical fields with node spacing based on Gauss-Lobatto-Legendre integration. This avoids Runge phenomena for basis functions of large polynomial degree [21] and produces a nodal spectral-element representation $[15,16]$. In practice, polynomials of degree three through nine are used. Gauss-Legendre integration is applied for the Galerkin projections. The number of integration points is chosen to integrate the product of basis and test functions exactly for rectangular elements in Cartesian coordinates. In nonlinear simulations, higher-order numerical integration is used to avoid aliasing error during the projection of quadratic nonlinearities.

When solving (15a-c) or (16a-c) in weak form, the function space, $\mathbf{V}_{h, N, \mathrm{p}}$, for vector fields is unchanged from NIMROD's standard $C^{0}$ spectral-element representation. For $\mathbf{V} \quad \mathbf{V}_{h, N, p}$,

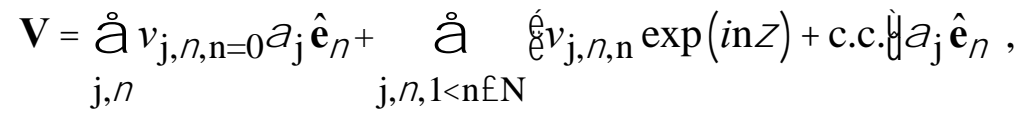

where $\mathrm{j}(1,2)$ are the spectral bases that are order $\mathrm{p}$ nodal polynomials of each of the two element coordinates ${ }_{1}$ and ${ }_{2}$, and c.c. indicates the complex conjugate of the preceding term. Coefficients for nodes located along element borders are unique, hence the $C^{0}$ continuity. Physical coordinates may be the cylindrical system $(R, Z$,$) , in which case =$, or a periodic Cartesian system $(x, y, z)$, where $\quad=2 \quad z / L_{z}$ for $0 \quad z \quad L_{z}$. The unit-direction vectors $\hat{\mathbf{e}}$ follow from the coordinate choice. Isoparametric mappings relate the element coordinates to physical coordinates $(x, y)$ or $(R, Z)$ in a non-overlapping partition, indicated by $h$, of the 2D cross section of the domain. For simplicity of presentation, homogeneous essential conditions are imposed on the surface-normal component of vector fields in $\mathbf{V}_{h, N, \mathrm{p}}$ along the entire boundary of the domain.

The new auxiliary projections for flow-divergence and parallel vorticity avoid duplication of the physical responses at resolved scales by using a function space, $F_{h, N \text {,p }}$, that is an incomplete polynomial expansion. For $f \quad F_{h, N, \mathrm{p}}$,

$$
f=f_{\mathrm{j}, \mathrm{n}=0} \mathrm{j}_{\mathrm{j}, 1<\mathrm{n} N} f_{\mathrm{j}, \mathrm{n}} \exp (\text { in })+\text { c.c. } \quad \mathrm{j},
$$

with 


$$
\beta_{j}\left(\xi_{1}, \xi_{2}\right) \in\left\{\bigcup_{l=0}^{\mathrm{p}} P_{l}\left(\xi_{1}\right) P_{\mathrm{p}}\left(\xi_{2}\right) \cup \bigcup_{l^{\prime}=0}^{\mathrm{p}-1} P_{\mathrm{p}}\left(\xi_{1}\right) P_{l^{\prime}}\left(\xi_{2}\right)\right\}
$$

in each element, where $P_{l}(\quad)$ is a Legendre polynomial. As an example, the set of ${ }_{j}(1,2)$ for $\mathrm{p}=3$ is plotted in Fig. 1. Unlike the ${ }_{j}$ bases, the ${ }_{j}$ functions are not continuous across element borders.

The weak form of the semi-discrete problem for evolving the flow velocity with auxiliary hyperbolic responses, Eqs. (15a-c), is then to find $\mathbf{v} \quad \mathbf{V}_{h, N, \mathrm{p}}$ and $\quad, \quad F_{h, N, \mathrm{p}}$ such that

$$
\begin{aligned}
& \int d V o l\left[\mathbf{W} \cdot \frac{\partial}{\partial t} \mathbf{v}+\frac{\partial}{\partial t}+\frac{\partial}{\partial t}\right]=\int d V o l\left[\mathbf{W} \cdot(\nabla \times \mathbf{b}) \times \mathbf{B}_{0}+\mathbf{W} \cdot \mathbf{J}_{0} \times \mathbf{b}\right] \\
& +\int d \operatorname{Vol}\left[p \nabla \cdot \mathbf{W}+f_{d} \sqrt{P_{c}}\left(\begin{array}{cc}
\nabla \cdot \mathbf{v} & \nabla \cdot \mathbf{W}
\end{array}\right)+f_{v} \mathbf{B}_{0} \cdot\left(\begin{array}{ll}
\nabla \times \mathbf{v} & \nabla \times \mathbf{W})
\end{array}\right]\right.
\end{aligned}
$$

for all $\mathbf{W} \quad \mathbf{V}_{h, N, \mathrm{p}}$ and,$\quad F_{h, N, \mathrm{p}}$. It is straightforward to generalize Eq. (19) for the nonlinear non-ideal system (1-5), where only the relatively large, spatially symmetric parts of $\mathbf{B}$ and $P$ are needed in the coefficients of the projection and response terms. The stabilizing aspect of the auxiliary responses can be inferred by taking $\mathbf{v} \rightarrow \partial \xi / \partial t$ and noting that the projections tend to make $\sigma \sim f_{d} \overline{\sqrt{P_{c}} \nabla \cdot \xi}$ and $\lambda \sim f_{v} \overline{\mathbf{B}_{0} \cdot \nabla \times \xi}$, where the bar indicates the projection onto $F_{h, N, \mathrm{p}}$. Substituting these relations in (19) leads to the symmetric terms $-f_{d}^{2} \overline{\left(\sqrt{P_{c}} \nabla \cdot \xi\right)} \overline{\left(\sqrt{P_{c}} \nabla \cdot \mathbf{W}^{*}\right)}$ and $-f_{v}^{2} \overline{\left(\mathbf{B}_{0} \cdot \nabla \times \xi\right)} \overline{\left(\mathbf{B}_{0} \cdot \nabla \times \mathbf{W}^{*}\right)}$ for the scales of the auxiliary projections. Similar to the penalty term for eigenvalue computations [4], they promote stable oscillatory behavior.

The weak form of the stabilized semi-discrete problem for evolving the flow velocity with auxiliary parabolic responses, Eqs. (16a-c), is to find $\mathbf{v} \quad \mathbf{V}_{h, N, \mathrm{p}}$ and,$\quad F_{h, N, \mathrm{p}}$ such that

$$
\begin{aligned}
& \int d V o l\left[\mathbf{W} \cdot \frac{\partial}{\partial t} \mathbf{v}++\right]=\int d V o l\left[\mathbf{W} \cdot(\nabla \times \mathbf{b}) \times \mathbf{B}_{0}+\mathbf{W} \cdot \mathbf{J}_{0} \times \mathbf{b}\right]
\end{aligned}
$$

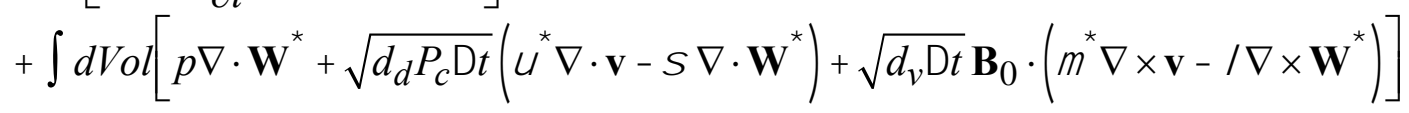

for all $\mathbf{W} \quad \mathbf{V}_{h, N, \mathrm{p}}$ and,$\quad F_{h, N, \mathrm{p}}$. Here, the responses effect viscous diffusion that targets interchange behavior at the scales of the auxiliary projections.

Because the ${ }_{j}$ bases have the Legendre polynomial of highest order in at least one of the two element coordinates, they are orthogonal to the larger scales on a given mesh. This keeps the auxiliary projections from interfering with physical responses to compression and parallel vorticity at spatially resolved scales with either the hyperbolic method or the parabolic method. The influence of the auxiliary projections also diminishes with increasing resolution without scaling their numerical parameters. In this sense the auxiliary projections and responses are similar to spectral filtering that has been developed for incompressible fluid modeling [7] and for other applications [22]. 
When either (19) or (20) is solved with implicit temporal differencing in time-dependent computations, the expansion coefficients of $\sigma$ and $\lambda$ at the end of a time-step are eliminated algebraically through matrix partitioning, i.e. "static condensation," for each element. Matrix partitioning is also used in the eigenvalue computations with parabolic responses that are described in Sect. 4. For both types of computations, the symmetric contributions from the projections appear in the Schur complement matrix, which has no loss of sparsity, owing to the discontinuous nature of the expansions for $\sigma$ and $\lambda$.

\subsection{Alternative magnetic-divergence control}

The eigenvalue results presented in Sect. 4 show that stability to numerical interchange is sensitive to the numerical treatment of magnetic divergence, in addition to nodal-scale responses to compression and parallel vorticity. Thus, alternative approaches to enforcing the magnetic divergence constraint are considered. NIMROD computations typically use the diffusive form of error control, $\quad={ }_{b} \nabla \cdot \mathbf{B}$, in Eq. (4); tests showing its effectiveness with high-order elements are described in [17]. In the present study, greater numerical flexibility is obtained by incorporating another auxiliary equation. We may take $\rightarrow+{ }_{b} \nabla \cdot \mathbf{B}$ in Eq. (4) with

$=d_{b} \nabla \cdot \mathbf{B}$, or with $\mathbf{b}$ in place of $\mathbf{B}$ for Eq. (9), as the auxiliary equation. For the eigenmode results presented in Sect. 4 , we set $\quad b \quad 0$ and $d_{b}=0$ or vice versa so that only one of the two parabolic methods for magnetic divergence control is used at a time. The hyperbolic response to divergence error, described in Ref. [19], is an alternative that can avoid additional numerical dissipation. In this method, $\rightarrow f_{b}$ is obtained by solving the auxiliary equation $\partial / \partial t=f_{b} \nabla \cdot \mathbf{B}$.

Unlike the novel projections for flow divergence and parallel vorticity described above, the auxiliary scalar $\phi$ for the magnetic divergence constraint is expanded in discontinuous polynomials that are complete through degree $\mathrm{p} 1$. Controlling divergence with an auxiliary field expanded in discontinuous bases of lower polynomial degree than the continuous expansion for the vector field is frequently used in incompressible fluid simulations [for example, $16,23,24]$. Whether using either the hyperbolic method or the parabolic method with $b=0$, the function space $C_{h, N, \mathrm{p}-1}$ for the auxiliary field is the same. For $\quad C_{h, N, \mathrm{p}-1}$,

$$
=\mathrm{j}_{\mathrm{j}, \mathrm{n}=0} \mathrm{j}_{\mathrm{j}, 1<\mathrm{n} N}^{+} \mathrm{j,n} \exp (\text { in })+\text { c.c. } \quad \mathrm{j},
$$

where $j(1,2)$ are the $2 \mathrm{D}$ modal Legendre bases that are discontinuous at element borders. The semi-discrete weak form of (9) with the hyperbolic constraint method is then to find b $\quad \mathbf{V}_{h, N, \mathrm{p}}$ and $\quad C_{h, N, \mathrm{p}-1}$ such that

$$
\int d \operatorname{Vol}\left[\mathbf{A} \cdot \frac{\partial}{\partial t} \mathbf{b}+\frac{\partial}{\partial t}\right]=\int d V o l\left[\nabla \times \mathbf{A} \cdot \mathbf{v} \times \mathbf{B}_{0}+f_{b}\left(\begin{array}{ll}
\nabla \cdot \mathbf{b} & \nabla \cdot \mathbf{A})
\end{array}\right]\right.
$$

for all $\mathbf{A} \quad \mathbf{V}_{h, N, \mathrm{p}}$ and $\quad C_{h, N, \mathrm{p}-1}$. The alternative weak form allowing either parabolic method of divergence control is to find $\mathbf{b} \quad \mathbf{V}_{h, N, \mathrm{p}}$ and $\quad C_{h, N, \mathrm{p}-1}$ such that 


$$
\begin{aligned}
\int d V o l\left[\mathbf{A} \cdot \frac{\partial}{\partial t} \mathbf{b}+\quad\right] & =\int d \operatorname{Vol}\left[\nabla \times \mathbf{A} \cdot \mathbf{v} \times \mathbf{B}_{0} \quad b(\nabla \cdot \mathbf{A})(\nabla \cdot \mathbf{b})\right] \\
& +\int d \operatorname{Vol}\left[\sqrt{d_{b}}\left(\begin{array}{rl}
\nabla \cdot \mathbf{b} & \nabla \cdot \mathbf{A})
\end{array}\right]\right.
\end{aligned}
$$

for all $\mathbf{A} \quad \mathbf{V}_{h, N, \mathrm{p}}$ and $\quad C_{h, N, \mathrm{p}-1}$.

\section{Eigenvalue computations}

Numerical eigenvalue computations in periodic cylindrical geometry provide a means to test auxiliary projections/responses and other stabilization methods. Neither cylindrical geometry nor empirical evaluation are sufficient for proving stability to numerical interchange in all timedependent computations, but they provide insight. Eigenvalue computations for an inhomogeneous equilibrium profile and for a homogeneous profile are presented in separate subsections after a discussion of the eigenvalue computations, themselves.

\subsection{Formulation of eigenvalue computations}

The eigenvalue computations use a numerical representation for the radial coordinate $r$ and $\exp [i \mathrm{~m}+i \mathrm{kz}]$ dependence in the periodic azimuthal and axial coordinates for a single $(\mathrm{m}, \mathrm{k})$ pair in a given computation. For the radial coordinate, components of a system of dependent fields are expanded in a 1D mesh of continuous or discontinuous nodal spectral elements or in global orthogonal polynomials over a single element. The code that generates the algebraic systems, CYL_SPEC, is typically run with the Chebyshev polynomial basis. Legendre polynomials may be used, but only very small quantitative changes result. The basis functions for the auxiliary flow-divergence and parallel-vorticity projections described in Sect. 3 amount to a single polynomial in each CYL_SPEC element. However, the bases for the auxiliary projections of magnetic divergence have more than one polynomial for $\mathrm{p}>1$.

Reference computations for the two equilibrium profiles solve Eq. (10) with the displacement components

$$
=r \operatorname{Re}(r),=\frac{\operatorname{Im}() B_{0_{z}} \operatorname{Im}(z) B_{0}}{B^{2}},=\frac{\operatorname{Im}(\quad) B_{0}+\operatorname{Im}\left(z_{z}\right) B_{0_{z}}}{B^{2}}
$$

such that

$$
\xi(r, \theta, z)=\left(\frac{\mathrm{X}}{r} \hat{\mathbf{e}}_{r}-i \Lambda \hat{\mathbf{e}}_{r} \times \mathbf{B}_{0}+i \mathrm{Z} \mathbf{B}_{0}\right) \exp [i \mathrm{~m} \theta+i \mathrm{k} z]+\text { c.c. },
$$

which reflects an arbitrary choice of phase for this system. Only $\mathrm{X}$ is differentiated with respect to $r$ in the ideal-MHD energy computations. Similar to the pioneering work of Ref. [13], continuous expansions of $\mathrm{X}$ with discontinuous expansions of $\Lambda$ and $\mathrm{Z}$ of polynomial degree reduced by 1 allow displacements with vanishing divergence and vanishing perpendicular divergence within each element if factors from geometry and $\mathbf{B}_{0}$ are treated as constants in each element. This expansion also conforms to the function space of the analytical variational problem. The algebraic system resulting from this expansion is real and symmetric, and ideal 
MHD has a bounded minimum eigenvalue ${ }^{2}$ [12], so a shift can be imposed to make the generalized eigenvalue system positive. CYL_SPEC then solves the generalized eigenvalue problem with LAPACK's [25] DSPGVD routine. In discussion that follows, Eq. (10) with $\xi$ expressed in $\mathrm{X}, \Lambda$, and $\mathrm{Z}$ is labeled the "X, $\Lambda, \mathrm{Z}$-system."

Eigenvalue computations for testing numerical stabilization methods in time-dependent modeling are applied to the first-order $\mathbf{v}, \mathbf{b}, p$-system, Eqs. $(6,8$, and 9), with $\partial / \partial t \rightarrow i$ and frequency $\omega$ is the eigenvalue. The continuity equation is not required in computations for static equilibria without gravity. Like the NIMROD implementation, the expansions for this system do not satisfy $\nabla \cdot \mathbf{b}=0$ identically. With the hyperbolic interchange stabilization method (19) and the hyperbolic magnetic divergence control (22), the expansions can be arranged into a real algebraic system, which again represents a choice of phase. The expansions have coefficients for the real parts of $v_{r}, b_{r}, \sigma$, and $\lambda$ and for the imaginary parts of $v, v_{z}, b, b_{z}, p$, and $\phi$. The eigenvalue computations for these systems are solved with the DGGEV routine, and they necessarily have numerical modes that are not in the analytical ideal-MHD model (6-9) with $\nabla \cdot \mathbf{b}=0$ and $=0$. In contrast, eigenvalue computations with parabolic stabilization and divergence control have complex eigenvalues, whereas without equilibrium flow, the analytical model has only purely real and purely imaginary frequencies. CYL_SPEC calls the ZGGEV routine to solve this system.

\subsection{Inhomogeneous profile and reference computations}

The first equilibrium profile is an inhomogeneous profile from Ref. [3], where the Suydam stability parameter has a simple form. The axial component of $\mathbf{B}_{0}$ is unity across the domain $0 \quad r$, and with $0=1, c_{A}=B_{0}(0) / \sqrt{0}$ is unity. The magnetic winding parameter or "safety factor," $q(r)=2 \quad r B_{0_{z}} / L_{z} B_{0}$ for cylindrical geometry, is

$$
q(r)=2 \frac{1+c_{2}^{2} r^{2}}{L_{z} c_{1}}
$$

which increases monotonically, as in a tokamak, and the $\mathbf{k} \times \mathbf{B}_{0}=0$ resonance condition is $q=2 \mathrm{~m} / \mathrm{k} L_{z}$ for the $(\mathrm{m}, \mathrm{k})$ harmonic. The pressure profile is obtained from radial force balance, and the Suydam parameter for this configuration is

$$
D_{s}(r) \quad \frac{1}{r B_{0_{z}}} \frac{q}{d q / d r} \div \frac{d P}{d r} \div=\frac{c_{1}^{2}}{\left(1+c_{2}^{2} r^{2}\right) c_{2}^{4} r^{2}}
$$

The two parameters are set as $c_{1}=4 / 7$ and $c_{2}=10 / 7$ in the following tests, which makes

$2 P_{0}(0) / B_{0}^{2}(0)=0.14$ and places the marginal interchange stability condition at $r=0.466$. Analytically, interchange instability exists for any harmonic that is resonant inside $r=0.466$. Two $\mathrm{m}=4$ harmonics are considered. The $\mathrm{k}=1.5$ harmonic has $D_{s}=0.200$ at its resonance, 
$r=0.507$, and is stable. The $\mathrm{k}=1.784$ harmonic has $D_{s}=0.443$ at its resonance, $r=0.371$, and is unstable.

Computations with the reference $\mathrm{X}, \Lambda, \mathrm{Z}$-system and low-order polynomials converge reliably but slowly from the stable side, as described for conforming computations in Refs. [3,4]. The low-order expansions are continuous linear elements for $\mathrm{X}$ and piecewise constants for $\Lambda$ and Z. Results for the least-stable modes of the two $m=4$ harmonics are shown in Fig. 2a, where the computed eigenvalue $\quad 2$ is plotted for meshes that range from 40 to 2560 elements. At low resolution, the $\mathrm{k}=1.784$ computations do not identify a growing mode, and at higher resolution, the converged growth rate, $\operatorname{Im}(\quad)>0$, is approached from the stable side. The $\mathrm{k}=1.5$ computations also find ${ }^{2}$ decreasing with increasing resolution, but 2 remains positive. The nearly singular nature of the differential equation is evident from the numerical eigenmode for the $\mathrm{k}=1.784, N_{e l}=640$ computation, which is shown in Fig. 2b. The large component in the $\hat{\mathbf{e}}_{r} \quad \mathbf{B}_{0}$ direction shows the dominance of rotation about $\mathbf{B}_{0}$, i.e. parallel vorticity. The eigenmode for the stable $\mathrm{k}=1.5$ harmonic has a similar shape, but it is yet more localized near its resonance.

\subsection{Results without flow stabilization}

Eigenmode results for the first-order $\mathbf{v}, \mathbf{b}, p$-system that are computed using the parabolic magnetic divergence control with $\quad b \quad 0$ and $d_{b}=0$ without the new flow-divergence and parallel-vorticity projections are representative of standard time-dependent computations with NIMROD. The trace with filled black squares in Fig. $3 \mathrm{a}$ for $\mathrm{k}=1.784$ shows growth rates of the fastest-growing mode, computed with continuous elements of polynomial degree 4 (quartic). The numerical $b$ parameter is set to 100 in these cases, but results are fairly insensitive to the value of ${ }_{b}$; with $N_{e l}=40, \operatorname{Im}()$ changes by $1.3 \%$ as $\quad b$ is varied from 1 to $10^{6}$. Convergence is from the unstable side as the number of elements is increased. The fastest mode in each computation has $|\operatorname{Re}(\quad)|<<\operatorname{Im}(\quad)$, but overstable modes also exist. Computations of the analytically stable $\mathrm{k}=1.5$ harmonic also find growing numerical modes, as shown in Fig. $3 \mathrm{~b}$. The numerical eigenfunctions for both harmonics at low resolution and for the $\mathrm{k}=1.5$ harmonic with all meshes are noisy, as illustrated in Fig. 3c. The cylindrical unit vectors are not aligned with the $\mathbf{B}_{0}$ and $\hat{\mathbf{e}}_{r} \quad \mathbf{B}_{0}$ directions. However, other CYL_SPEC computations with field-aligned components for the first-order system (not shown) also converge from the unstable side.

The remaining three sets of eigenmode computations shown in Figs. 3a-b test other approaches for responding to divergence in $\mathbf{b}$ and $\mathbf{v}$. Results labeled with $d_{b}=100$ use a piecewise cubic auxiliary scalar $\phi$ with the parabolic response to $\nabla \cdot \mathbf{b}$. Results labeled with $f_{b}^{2}=100$ use the piecewise cubic auxiliary scalar $\phi$ with the hyperbolic response to $\nabla \cdot \mathbf{b}$. Both sets find numerical modes that grow at increasingly fast rates as the number of elements is increased. The fastest-growing modes are noisy and concentrated near $r=0$ with excursions in $\phi$. The discontinuous representation for $\phi$ does not satisfy the mathematical regularity condition 
of $\rightarrow 0$ for $r \rightarrow 0$. Using a nodal expansion of $\phi$ based on Radau integration points in the first element places a node at $r=0$, so that regularity can be imposed. However, with this modification, the fastest growing mode for the 80-element $\mathrm{k}=1.784$ computation with hyperbolic divergence response, for example, has a similar eigenfunction, apart from $(0)=0$, and it grows slightly faster. Yet another computation has equal-area meshing to improve the relative scaling of matrix elements from across the domain. Here, the most-unstable mode is in the middle of the domain, but it is also noisy.

The last set of results shown in Figs. 3a-b has the hyperbolic magnetic divergence response, and the pressure equation is modified to find the total perturbed pressure $p_{T}=\mathbf{B}_{0} \times \mathbf{b}+p$. The Lorentz force density in Eq. (6) is also reorganized into tensile, $\mathbf{B}_{0} \cdot \nabla \mathbf{b}+\mathbf{b} \cdot \nabla \mathbf{B}_{0}$, and compressive parts, allowing a separate numerical expansion of the total pressure. Like $\phi, p_{T}$ is expanded as a discontinuous cubic. This formulation is related to methods used for incompressible fluid computations for flows with viscosity [16,24]. However, it has difficulty reproducing the physically stabilizing responses when misapplied to this ideal compressible system.

With the flexibility of the spectral elements and the intended application to weakly dissipative systems, scanning the degree of the polynomial bases is also of interest. Results from computations using the parabolic $\nabla \cdot \mathbf{b}$ control with $\quad b \quad 0$ and $d_{b}=0$, i.e. using the $\nabla \nabla \cdot \mathbf{b}$ term, with continuous expansions of $\mathbf{v}, \mathbf{b}$, and $p$ of equal order exhibit similar behavior (Fig. 4) as the computations with quartic elements where the number of elements is scanned (Fig. 3). The $\mathrm{k}=1.784$ computations converge slowly on the unstable mode, while the $\mathrm{k}=1.5$ results have difficulty avoiding growth. In general, the $p$-refinement approach is not well suited to the nearly singular behavior of ideal interchange. However, the results show that it does not present additional problems relative to grid-based resolution.

\subsection{Results with flow stabilization}

The computations described above establish the need for stabilizing numerical interchange with the $C^{0}$ representation for the first-order system, and they provide a baseline for comparing results with the new projections. The low-resolution $N_{e l}=20$ computations of Figs. 3a-b are the most problematic ones when using the parabolic $\nabla \nabla \cdot \mathbf{b}$ control. They are also convenient for scanning the normalized $d_{d}$ and $d_{v}$ parameters for the diffusive projections (with $\Delta t$ in Eq. (20) set to unity) and the normalized $f_{d}$ and $f_{v}$ parameters for the hyperbolic projections.

Results of these scans for the auxiliary flow-divergence and parallel-vorticity projections are shown in Fig. 5. With the parabolic $\nabla \nabla \cdot \mathbf{b}$ control and the diffusive flow projections (Figs. 5ab), the additional responses are effective when both $d_{d}$ and $d_{v}$ parameters are sufficiently large. When the magnetic divergence control is through the auxiliary $\phi$ expansion, both the parabolic (Figs. 5c-d) and the hyperbolic (Figs. 5e-f) responses reduce unresolved growth when either the flow-divergence or the parallel-vorticity parameter is sufficiently large. When using the auxiliary $\phi$ expansion for controlling $\nabla \cdot \mathbf{b}$, the behavior of the two forms of the auxiliary responses is similar. One difference is that complete stabilization is observed in these lowresolution computations with hyperbolic responses; all modes are purely oscillatory when $f_{d}$ 
and $f_{v}$ are large. In contrast, the results with parabolic responses and large $d_{d}$ and $d_{v}$ parameters in Figs. (5c-d) have slowly growing modes with $\operatorname{Im}() \sim 10^{5}$, which is approximately three orders of magnitude smaller than growth rates from computations without the flow-divergence and parallel-vorticity projections. Computations in the cyan region of Figs. 5a-b, which use the parabolic $\nabla \nabla \cdot \mathbf{b}$ control, have growing modes with $\operatorname{Im}(\quad) 10^{4}$, despite the larger $d_{d}$ and $d_{v}$ parameters.

The 20-element results of Fig. 5 are used to guide the selection of the normalized parameters for the flow-divergence and parallel-vorticity responses for convergence studies. Among the computations shown in Fig. 6, those using the parabolic $\nabla \nabla \cdot \mathbf{b}$ control have ${ }_{b}=100, d_{d}=100$ , and $d_{v}=1$; those using the auxiliary $\phi$ with parabolic projections have $d_{b}=100, d_{d}=10^{2}$, and $d_{v}=10^{4}$; and those with hyperbolic projections have $f_{b}^{2}=100, f_{d}^{2}=10^{2}$, and $f_{v}^{2}=10^{4}$ . The auxiliary projections make the computations of the unstable $\mathrm{k}=1.784$ harmonic converge from the stable side, as shown in Fig. 6a, which is an improvement relative to computations without projection. The sets of computations for the analytically stable $\mathrm{k}=1.5$ harmonic still find growing modes, as shown in Fig. 6b. Nonetheless, the two sets using the auxiliary $\phi$ have growth rates reduced by more than two orders of magnitude below the related computations which do not have the auxiliary flow-divergence and parallel-vorticity responses (Fig. 3b). Numerical interchange seems to be more resistant to these responses when using the parabolic $\nabla \nabla \cdot \mathbf{b}$ magnetic divergence control in place of either the hyperbolic or parabolic methods with the auxiliary $\phi$ expansion.

Eigenfunctions of the fastest-growing modes from the 40-element computations with hyperbolic projections are compared in Fig. 7. Although the growth rate of the $k=1.784$ mode is less than $50 \%$ of its converged value, the computed eigenfunction is already similar to the result for the $\mathrm{X}, \Lambda, \mathrm{Z}$-system shown in Fig. 2 . In contrast, the weakly growing $\mathrm{k}=1.5$ mode of Fig. $7 \mathrm{~b}$ has a noisy eigenfunction with a relatively large axial component. This is related to the fact that its eigenvalue has a large real part, $|\operatorname{Re}()| 1000 \operatorname{Im}()$. The other modes shown in Fig. 6b, including those that use the parabolic $\nabla \nabla \cdot \mathbf{b}$ control, also have $|\operatorname{Re}(\quad)| \gg>\operatorname{Im}(\quad)$. Besides these overstable modes, which are the fastest-growing modes from each computation, the numerical spectra have other growing modes with $|\operatorname{Re}(\quad)|<<\operatorname{Im}(\quad)$. Their growth rates are considerably smaller than those of the fastest-growing, overstable modes.

The projections for flow divergence and parallel vorticity are also intended for applying $p$ refinement. The projections use just the Chebyshev polynomial of degree $\mathrm{p}$ in each element, so it changes with increasing resolution. Results using the hyperbolic projections and responses for both $\mathrm{m}=4$ harmonics are presented in Fig. 8. As with mesh-based refinement for fixed $\mathrm{p}$, convergence on the unstable mode is from the stable side. While growth is observed for the analytically stable mode, the computed growth-rate of the fastest growing mode remains small over this scan.

\subsection{Results with a mixed-order representation}


A final set of eigenmode computations for this equilibrium considers an adaptation of the approach described in Ref. [6]. The computations use continuous expansions for $\mathbf{v}, \mathbf{b}$, and $p$ without flow-divergence and parallel-vorticity projection, and the polynomial degree of the expansion for $\mathbf{v}$ is reduced to $\mathrm{p}$ 1. Figure 9 shows the fastest growing mode for the two $\mathrm{m}=4$ harmonics. Clearly, convergence is from the stable side for the $\mathrm{k}=1.784$ mode. Moreover, with the hyperbolic method of controlling $\nabla \cdot \mathbf{b}$ error, there are no unstable modes for $\mathrm{k}=1.5$. Based on these test results, reducing the degree of the $\mathbf{v}$-expansion is very effective for controlling linear numerical interchange.

\subsection{Homogeneous-profile spectra}

The second cylindrical equilibrium profile is homogeneous with $\mathbf{B}_{0}=B_{0} \hat{\mathbf{z}}$ and uniform $P_{0}$ and 0 . There is no free energy, and eigenmode computations with this profile provide basic tests of stable waves. Setting $==1$ for $\mathrm{k}=2$ places the accumulation point of the sound spectrum at $\quad 2=4 / 3$, and the entire Alfvén spectrum of torsional waves is degenerate at

$2=4$. For $\mathrm{m}=1$, for example, the sound spectrum has a maximum at ${ }^{2}=1.550$, and the lowest-frequency magneto-acoustic fast wave is at ${ }^{2}=9.536$. Analytically, no modes exist for

$2<4 / 3$, for $1.550<2<4$, and for $4<2<9.536$. The sound and magneto-acoustic waves have radially smooth eigenfunctions. The torsional waves of the homogeneous equilibrium do not couple fluid elements over the radial coordinate, so their radial profiles are arbitrary. Thus, purely spectral computation is well suited to the nonsingular nature of this problem, and results from the $\mathrm{X}, \Lambda, \mathrm{Z}$-system with global Chebyshev polynomials of shifted quadratic argument [21], $T_{j}\left(\begin{array}{ll}2 r^{2} & 1\end{array}\right)$, are used for reference.

A comparison of results from three finite-element computations and the reference computation is shown in Fig. 10. Each element-based computation has four finite elements over the radial coordinate with $\mathbf{b}$ and $p$ expanded in continuous cubic polynomials. The spectrum on the right sides of the two frames is computed with a continuous quadratic expansion for $\mathbf{v}$, and the other two computations have cubic expansions for $\mathbf{v}$. All three use hyperbolic responses to $\nabla \cdot \mathbf{b}$ with the auxiliary $\phi$ expanded in discontinuous quadratics and $f_{b}^{2}=100$. Among the three finite-element computations, the equal-order representation without flow-divergence and parallel-vorticity projection is the best approximation of the reference. Like the analytical spectrum, it has three regions where eigenvalues do not exist. In contrast, the result with quadratic v-expansion has modes between the Alfvén spectrum and the first fast mode, and its sound spectrum extends above ${ }^{2}=1.550$. Modes in these regions represent forms of spectral pollution [3]. Apart from modes at ${ }^{2}=0$, results of the computation with flow-divergence ( $\left.f_{d}^{2}=10^{2}\right)$ and parallel-vorticity $\left(f_{v}^{2}=10^{4}\right)$ projection are similar to those of the equal-order expansion without these projections. With the projections, there are eight zero-frequency modes, which have either the $\sigma$-component or the $\lambda$-component of the eigenfunction being approximately two orders of magnitude or more larger than any of the physical components. This system admits a null space for these auxiliary fields, and its dimension is one per auxiliary 
field per element. Modes of this null space do not excite a response in the flow velocity, so they are not problematic for time-dependent computation. The computation with the quadratic vexpansion has twelve zero-frequency modes. In contrast to the equal-order computation with auxiliary flow-divergence and parallel-vorticity projection, the eigenfunctions are dominantly in components of $\mathbf{b}$, and $p$. This null space can be driven through physical nonlinear coupling in time-dependent computations.

\section{Time-dependent computations}

When incorporating the flow-divergence, parallel-vorticity, and magnetic-divergence projections in the NIMROD solver, there are additional considerations for the numerical timeadvance. The projections have been tested with the implicit leapfrog advance that is described in Ref. [26], as applied to MHD with implicit advection. Each physical field is advanced separately and implicitly, and to avoid time-step limitations, the implementation of the new projections is also implicit. For the magnetic-field advance with the auxiliary $\phi$ projection, for example, the temporally semi-discrete equation is

$$
\frac{\mathbf{B}^{n+1} \mathbf{B}^{n}}{t}=\nabla \times \mathbf{E}+{ }_{b} \nabla \nabla \cdot\left[{ }_{b} \mathbf{B}^{n+1}+(1 \quad b) \mathbf{B}^{n}\right]+\nabla
$$

where superscripts indicate the time-step indices, $\quad b$ is a centering parameter,

$$
\begin{aligned}
& =f_{b} t\left\lfloor b^{n+1}+\left(\begin{array}{ll}
1 & b
\end{array}\right)^{n}\right\rfloor, \\
& { }^{n+1} \quad n=f_{b} t \nabla \cdot\left[{ }_{b} \mathbf{B}^{n+1}+(1 \quad b) \mathbf{B}^{n}\right]
\end{aligned}
$$

for the hyperbolic method, and

$$
=d_{b} t \nabla \cdot\left\lfloor{ }_{b} \mathbf{B}^{n+1}+\left(\begin{array}{ll}
1 & b
\end{array}\right) \mathbf{B}^{n}\right\rfloor
$$

for the parabolic method. The implementations of the flow-divergence and parallel-vorticity projections are similar with centering parameter $v$. During each step, the updated $\phi$ and $\mathbf{B}$ coefficients are solved simultaneously, and the updated $\sigma, \lambda$, and $\mathbf{V}$ coefficients are solved simultaneously.

The same class of inhomogeneous cylindrical equilibria from Ref. [3] considered in Sect. 4 also provides convenient tests for our time-dependent computations. The reported growth rates are inferred from the evolution of computed energy after a dominant mode is obtained. To best represent full-scale toroidal simulations, the 1D finite Fourier series is used for the $z$-coordinate, and a circular-polar mesh of curved $2 \mathrm{D}$ elements is used for the $r-\theta$ plane. The circular-polar mesh is aligned with the equilibrium. However, vector expansions in this representation use Cartesian unit vectors, so the distinct radial and azimuthal components of interchange modes are mixed algebraically.

A general observation from running linear time-dependent computations with the new projections is that time-centered $(v=1 / 2)$ hyperbolic projections for $\mathbf{V}$ are not effective at 
stabilizing numerical interchange in NIMROD. This is counter to expectations from the CYL_SPEC results shown in Fig. 6 with hyperbolic projections, but the numerical instabilities are noisy in both meshed coordinates, which is not tested by single- $(\mathrm{m}, \mathrm{k})$ eigenmode computations. Projection with $v>1 / 2$ is more effective. An example that demonstrates this has $c_{1}=1 / 2, c_{2}=3 / 2$, and $\mathrm{k}=1.1$, which makes $D_{s}=0.224$ at the $\mathrm{m}=3$ resonance. A computation with $v_{v}=f_{d}=f_{v}=1$, a biquartic 1212 mesh, and $t=5$ (in Alfvén times, $A_{A}$ ) does not produce a growing mode over 18,000 time-steps, while changing $v$ to $1 / 2$ produces a noisy numerical mode that grows at $3.110^{3}$. Setting $v_{v}>1 / 2$ introduces numerical dissipation from the implicit projections, and that is beneficial. Results with the hyperbolic and parabolic projections at $\quad v=1$ are very similar, and both projection methods are able to avoid growing numerical modes in the analytically stable $\mathrm{k}=1.1$ case.

Convergence of time-dependent linear computations is tested with the physically unstable $\mathrm{m}=4, \mathrm{k}=1.784$ calculation described in Sect. 4. Figure 11 demonstrates that the flowdivergence and parallel-vorticity projections, using ${ }_{v}=d_{d}=1$ and $d_{v}=0.1$, effect convergence from the stable side for these computations. This is achieved with the $\nabla \nabla \cdot \mathbf{B}$ divergence control $\left({ }_{b}=0.01\right.$ and $\left.d_{b}=0\right)$ and with the discontinuous bicubic $\phi$ expansion $\left({ }_{b}=0\right.$ and $\left.d_{b}=1\right)$. Scaling the numerical parameters for the flow stabilization shows that there is some sensitivity when the physical mode is not resolved. For example, scaling $d_{d}$ keeping $d_{v} / d_{d}=0.1$ with the 4824 mesh of quartic elements and the discontinuous bicubic $\phi$ expansion finds less than $1 \%$ variation in $\operatorname{Im}()$ over $10^{3} d_{d} \quad 0.1$. For larger values of $d_{d}, \operatorname{Im}()$ drops more appreciably from $5.5310^{3}$ at $d_{d}=1$ to approximately $2 \quad 10^{3}$ at $d_{d}=100$ and to $510^{4}$ at $d_{d}=10^{3}$. However, the impact of the auxiliary responses diminishes rapidly with increasing spatial resolution. With the 9648 mesh, there is less than $1 \%$ variation in $\operatorname{Im}(\quad)$ over $10^{3} d_{d} 10^{3}$.

To check whether the new projections improve convergence in toroidal geometry, linear computations of ballooning-dominated edge-localized modes (ELMs) have been performed. The circular cross-section cbm18 equilibrium, case 2 of Ref. [27], is used. The computations have small uniform physical dissipation parameters such that $\mathrm{S} \quad r / A^{\sim 1 /}=10^{10}$ with viscous, thermal, and particle diffusivities having the same small values as the magnetic diffusivity. The results shown in Fig. 12 are from computations that scan azimuthal resolution, keeping the nonuniform radial meshing fixed, with and without the flow-divergence and parallel-vorticity projections. All of the computations use the $\nabla \nabla \cdot \mathbf{B}$ control, but only those shown in Fig. 12b have the projections and use $d_{d}=0.5$ and $d_{v}=0.1$. Similar to the findings on cylindrical interchange, the projections make the numerical representation of ballooning converge from the stable side.

A final comparison with the inhomogeneous cylindrical equilibrium investigates the utility of the projections in nonlinear computation. Setting $c_{1}=4 / 7, c_{2}=10 / 7$, and $L_{z}=4 / 3$ places the resonances of the $(3,1)$ and $(7,2)$ harmonics in the unstable region. The nonlinear 
computations with and without the projections are non-ideal: $=10^{8}, \mathrm{Pm}=/=10$, $D_{n}=10^{8}$, and $=10^{7}$, where $v$ and $\chi$ are the viscous and thermal diffusivities. A 3236 mesh of biquintic elements represents the $r$ - $\theta$ plane, and the Fourier expansion for the axial coordinate has $0 \quad \mathrm{n} \quad 21$. The two computations use the $\nabla \nabla \cdot \mathbf{B}$ control with $\quad b=0.01$, and in the computation with projections, the parameters are $d_{d}=1$ and $d_{v}=0.3$. NIMROD determines the evolution of nonlinear perturbations about a prescribed steady state, so there is an effective drive that attempts to maintain the original equilibrium.

The computation with projections is able to proceed past the point where the nonlinear interchange ceases to remain laminar. The three plots in Fig. 13 show slices of the 3D pressure distribution just before the transition, just after, and before the start of another large-amplitude event. After the first transition, the simulation dynamics represent turbulent transport of thermal energy. The nonlinear computation without the projections is not able to proceed through the first transition. The decomposition of kinetic energies in Fig. 14b shows that the short wavelengths accumulate noise, which ends the computation, unlike the behavior with projections (Fig. 14a).

\section{Discussion and conclusions}

To recap the development and findings, the auxiliary numerical projections for numerical interchange are based on the how the stabilizing but singular magnetic-field bending response is realized in first-order compressible MHD systems. The expansions for the auxiliary projections of flow divergence and parallel vorticity are intentionally incomplete. Physically representative responses are in the system of equations for the physical fields, and the incomplete projections add coercive terms for the shortest wavelengths of a finite-element or spectral-element mesh, where physical responses are not modeled accurately. Results from 1D eigenmode computations and from time-dependent linear computations with 2D meshing show that the projections can modify the numerical realization of ideal resonant interchange instabilities to converge from the stable side. The projections also reliably reduce the growth rates of numerical instabilities by orders of magnitude in physically stable conditions with bad curvature. As intended, the projections improve the robustness of nonlinear MHD simulations. This is demonstrated by the cylindrical computations in Sect. 5, where the projections enable the computation to proceed into the phase of turbulent transport.

Most of the numerically unstable modes that survive projection in eigenmode computations for analytically stable conditions are overstable. In some of the time-dependent linear tests that find numerical instability in stable conditions, the growing mode is also overstable. With the auxiliary $\phi$ projection for controlling magnetic divergence, setting the centering parameter for both hyperbolic and parabolic projections to unity $(v=1)$ removes residual modes. Since some numerical dissipation is needed, and given the similarity of time-dependent results with parabolic and hyperbolic projection, the parabolic method is preferred. It does not retain the auxiliary $\sigma$ and $\lambda$ fields from time-step to time-step, and fewer algebraic computations are needed.

Use of the auxiliary $\phi$ projection of polynomial degree $\mathrm{p} \quad 1$ for controlling $\nabla \cdot \mathbf{B}$ instead of adding the $\nabla \nabla \cdot \mathbf{B}$ term directly in Faraday's law also helps suppress numerical interchange 
when the flow-divergence and parallel-vorticity projections are used. Although results are

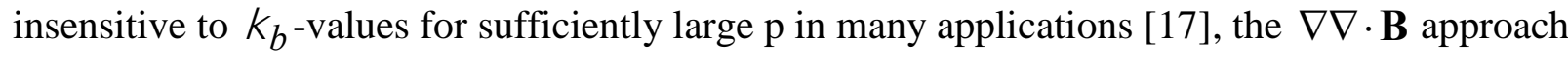
does not satisfy divergence stability for $C^{0}$ vector expansions [23]. The nominally irrotational and solenoidal parts of $C^{0}$ vector expansions retain some numerical coupling. This implies that the ${ }_{b} \nabla \nabla \cdot \mathbf{B}$ term can produce some level of numerical resistive dissipation, possibly allowing resistive interchange-like behavior when $=0$. The threshold for resistive interchange in cylindrical geometry is $D_{S}=0$ [28], so this effect adds to the tendency of numerical interchange in computations of ideal MHD.

The lack of distinct irrotational and solenoidal parts of $C^{0}$ vector expansions is also likely to affect the auxiliary responses for flow at low spatial resolution. The eigenvalue results shown in Figs. (5c-f) imply that numerical interchange can be stabilized with either the auxiliary flowdivergence response alone or the auxiliary parallel-vorticity response alone, provided that magnetic divergence control is through the auxiliary $\phi$ field. This observation may not be representative of computations with either a finer mesh of elements or with basis functions of larger polynomial degree, where the representation of irrotational and solenoidal vectors improves. The analytical $g$-mode behavior implies that both flow-divergence and parallelvorticity responses are critical for realizing a non-trivial interchange threshold, and that is consistent with the author's findings in other computational tests. The low-resolution behavior may be the result of one projection inducing both responses through numerical coupling of irrotational and solenoidal dynamics.

Reducing the degree of the polynomial expansion for flow velocity is the best adaptation of the recommendation in Ref. [6] for NIMROD. Improvement on interchange without flowdivergence and parallel-vorticity projection is obtained in the eigenmode computations described in Sect. 4 and in time-dependent linear computations with NIMROD that are not described above. However, nonlinear results with the reduced $\mathbf{V}$-expansion in NIMROD prove to be less robust than standard equal-order computations. This is attributed to the zero-frequency linear modes with eigenfunctions having significant physical components. Nonlinear coupling appears to drive these linearly unresponsive shapes until a nonlinear numerical instability results. This behavior may be specific to representing the primitive $\rho, \mathbf{V}, \mathbf{B}$, and $T$ fields in NIMROD's $C^{0}$ representation. It is not a general property of mixed-order MHD computation; the conforming low-order eigenmode expansion of Refs. [3,13] is a counter-example. The choice of which variables are expanded also influences numerical interchange behavior. There are similarities in the temporal advances and element-based expansions of NIMROD and M3D-C1 [29], but the potential-based formulation of the M3D equations avoids numerical interchange without special treatment [30].

Returning to the topic of adding numerical dissipation, the parabolic flow-divergence and parallel-vorticity projections with $v=1$ are forms of numerical hyperviscosity. That they act through projections onto the orthogonal polynomial of largest degree in a computation makes them similar to the spectral filtering of Ref. [7]. However, they are specially tailored to reinforce the physical responses to flow divergence and parallel vorticity that stabilize resonant interchange at a finite level of bad magnetic curvature. When computations are numerically resolved, results are independent of the choice of parameters, as demonstrated through the wide 
variety of ways that the unstable $m=4$ cylindrical mode is reproduced in Sect. 4 . Deriving optimal parameters is beyond the scope of this work, but the utility of finding optimal parameters can be inferred from considering the nonlinear computation of Sect. 5. A convergence study has not been performed, and the impact of the projections on the turbulent cascading of energy is not yet known. That the projections improve robustness is beneficial for being able to perform the nonlinear computation, but additional guidance on the choice of parameters, including the scaling with $\Delta t$, would help make the best use of a given level of numerical resolution.

\section{Acknowledgements}

The author thanks Drs. Paul Fischer, Stephen Jardin, and Nathaniel Ferraro for discussions on various topics that are related to this effort. The author is also grateful to Dr. Jacob King and to the anonymous reviewers, whose comments have helped improve this article. This work is supported by the U.S. Department of Energy, Scientific Discovery through Advanced Computing award DE-FC02-08ER54975. The nonlinear and ELM computations presented in Sect. 5 used resources of the National Energy Research Scientific Computing Center, a DOE Office of Science User Facility supported by the Office of Science of the U.S. Department of Energy under Contract No. DE-AC02-05CH11231.

\section{References}

[1] B. R. Suydam, Stability of a linear pinch, in Proceedings of the Second United Nations International Conference on the Peaceful Uses of Atomic Energy, United Nations, Geneva, 1958, Vol. 31, pp. 157-159.

[2] C. Mercier, A necessary condition for the hydromagnetic stability of plasma with axial symmetry, Nucl. Fusion 1 (1960) 47-53.

[3] R. Gruber, J. Rappaz, Finite Element Methods in Linear Ideal Magnetohydrodynamics, Springer, Berlin, 1985, pp. 79-117.

[4] L.M. Degtyarev, S.Yu. Medvedev, Methods for numerical simulation of ideal MHD stability of axisymmetric plasmas, Comput. Phys. Commun. 43 (1986) 29.

[5] A. Bondeson, G. Y. Fu, Tunable integration scheme for the finite element method, Comput. Phys. Commun. 66 (1991) 167-176.

[6] H. Lütjens, J. F. Luciani, A class of basis functions for non-ideal magnetohydrodynamic computation, Comput. Phys. Commun. 95 (1996) 47-57.

[7] P. F. Fischer, J. S. Mullen, Filter-based stabilization of spectral element methods, C. R. Acad. Sci. Paris 332 (2001) 265-270.

[8] A. N. Brooks, T. J. R. Hughes, Streamline upwind/Petrov-Galerkin formulations for convection dominated flows with particular emphasis on the incompressible Navier-Stokes equations, Comput. Methods Appl. Mech. Engrg. 32 (1982) 199-259.

[9] L. P. Franca, G. Hauke, A. Masud, Revisiting stabilized finite element methods for the advection-diffusion equation, Comput. Methods Appl. Mech. Engrg 195 (2006) 1560-1572.

[10] J. N. Shadid, R. P. Pawlowski, J. W. Banks, L. Chacón, P. T. Lin, R. S. Tuminaro, Towards a scalable fully-implicit fully-coupled resistive MHD formulation with stabilized FE methods, J. Comput. Phys. 229 (2010) 7649-7671. 
[11] C. Canuto, Stabilization of spectral methods by finite element bubble functions, Comput. Methods Appl. Mech. Engrg. 116 (1994) 13-26.

[12] J. P. Freidberg, Ideal magnetohydrodynamic theory of magnetic fusion systems, Rev. Mod. Phys. 54 (1982) 801-902.

[13] K. Appert, D. Berger, R. Gruber, J. Rappaz, A new finite element approach to the normal mode analysis in magnetohydrodynamics, J. Comput. Phys. 18 (1975) 284-299.

[14] D. Berger, R. Gruber, F. Troyon, A finite element approach to the computation of the MHD spectrum of straight noncircular plasma equilibria, Comput. Phys. Commun. 11 (1976) 313323.

[15] A. T. Patera, A spectral element method for fluid dynamics: laminar flow in a channel expansion, J. Comput. Phys. 54 (1984) 468-488.

[16] M. O. Deville, P. F. Fischer, E. H. Mund, High-order methods for incompressible fluid flow, Cambridge Press, Cambridge, 2002, pp. 62-67, 238-245.

[17] C.R. Sovinec, A.H. Glasser, T.A. Gianakon, D.C. Barnes, R.A. Nebel, S.E. Kruger, D.D. Schnack, S.J. Plimpton, A. Tarditi, M.S. Chu, the NIMROD Team, Nonlinear magnetohydrodynamics simulation using high-order finite elements, J. Comput. Phys. 195 (2004) 355-386.

[18] S. Chandraseker, Hydrodynamic and Hydromagnetic Stability, Dover, Mineola, New York, 1981, 577-598.

[19] C.-D. Munz, R. Schneider, E. Sonnendrücker, U. Voß, Maxwell's equations when the charge conservation is not satisfied, C. R. Acad. Sci. Paris 328 (1999) 431-436; C.-D. Munz, P. Omnes, R. Schneider, E. Sonnendrücker, U. Voß, Divergence correction technique for Maxwell solvers based on a hyperbolic model, J. Comput. Phys. 161 (2000) 484-511.

[20] T. J. R. Hughes, W. K. Liu, and A. Brooks, Finite element analysis of incompressible viscous flows by the penalty function formulation, J. Comput. Phys. 30 (1979) 1-60, and references therein.

[21] J. P. Boyd, Chebyshev and Fourier spectral methods, 2nd ed., Dover, Mineola, New York, 2001, pp. 82-86, 385-389.

[22] J. P. Boyd, Two comments on filtering (artificial viscosity) for Chebyshev and Legendre spectral and spectral element methods: preserving boundary conditions and interpretation of the filter as a diffusion, J. Comput. Phys. 143 (1998) 283-288.

[23] J. M. Boland, R. A. Nicolaides, Stability of finite elements under divergence constraints, SIAM J. Numer. Anal. 20 (1983) 722-731.

[24] C. Bernardi, Y. Mayday, A collocation method over staggered grids for the Stokes problem, Int. J. Numer. Methods Fluids 8 (1988) 537-557.

[25] E. Anderson, Z. Bai, C. Bischof, S. Blackford, J. Demmel, J. Dongarra, J. Du Croz, A. Greenbaum, S. Hammarling, A. McKenney, D. Sorensen, LAPACK Users' Guide, 3rd ed., Society for Industrial Applied Mathematics, Philadelphia (1999).

[26] C. R. Sovinec, J. R. King, the NIMROD Team, Analysis of a mixed semi-implicit/implicit algorithm for low-frequency two-fluid plasma modeling, J. Comput. Phys. 229 (2010) 58035819. 
[27] B. J. Burke, S. E. Kruger, C. C. Hegna, P. Zhu, P. B. Snyder, C. R. Sovinec, E. C. Howell, Edge localized linear ideal magnetohydrodynamic instability studies in an extendedmagnetohydrodynamics code, Phys. Plasmas 17 (2010) 032103.

[28] B. Coppi, J.M. Greene, J.L. Johnson, Resistive instabilities in a diffuse linear pinch, Nucl. Fusion 6 (1966) 101.

[29] N. M. Ferraro, S. C. Jardin, Calculations of two-fluid magnetohydrodynamic axisymmetric steady states, J. Comput. Phys. 228 (2009) 7742-7770.

[30] S. C. Jardin and N. M. Ferraro, private communication, 2013. 

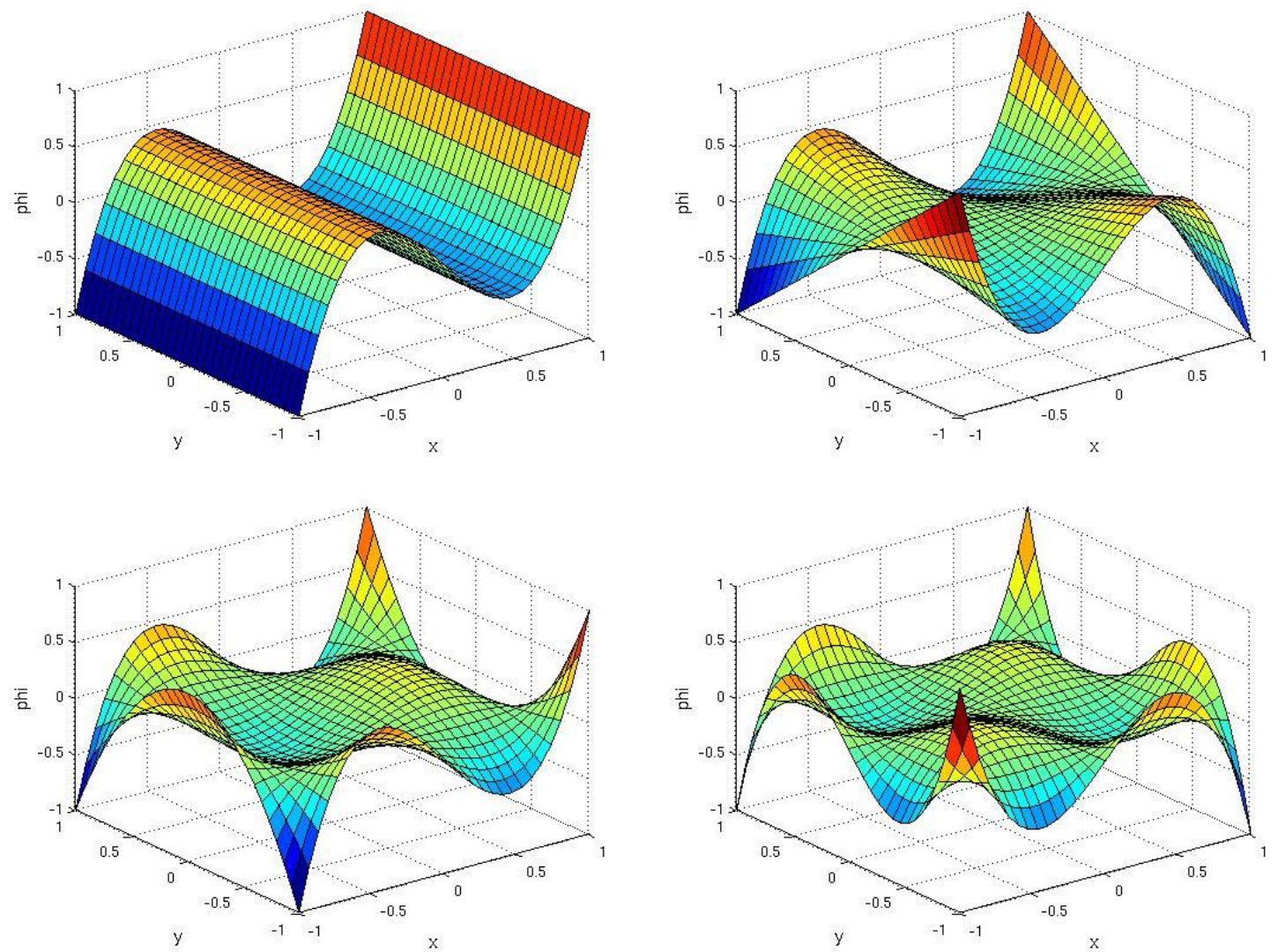

Figure 1. Illustration of four of the seven functions used for projecting $\sigma$ and $\lambda$ for $\mathrm{p}=3$, where the $x$ and $y$ axis labels refer to the ${ }_{1}$ and ${ }_{2}$ element coordinates in the text. The remaining three functions are the first three plots with $x$ and $y$ transposed. 

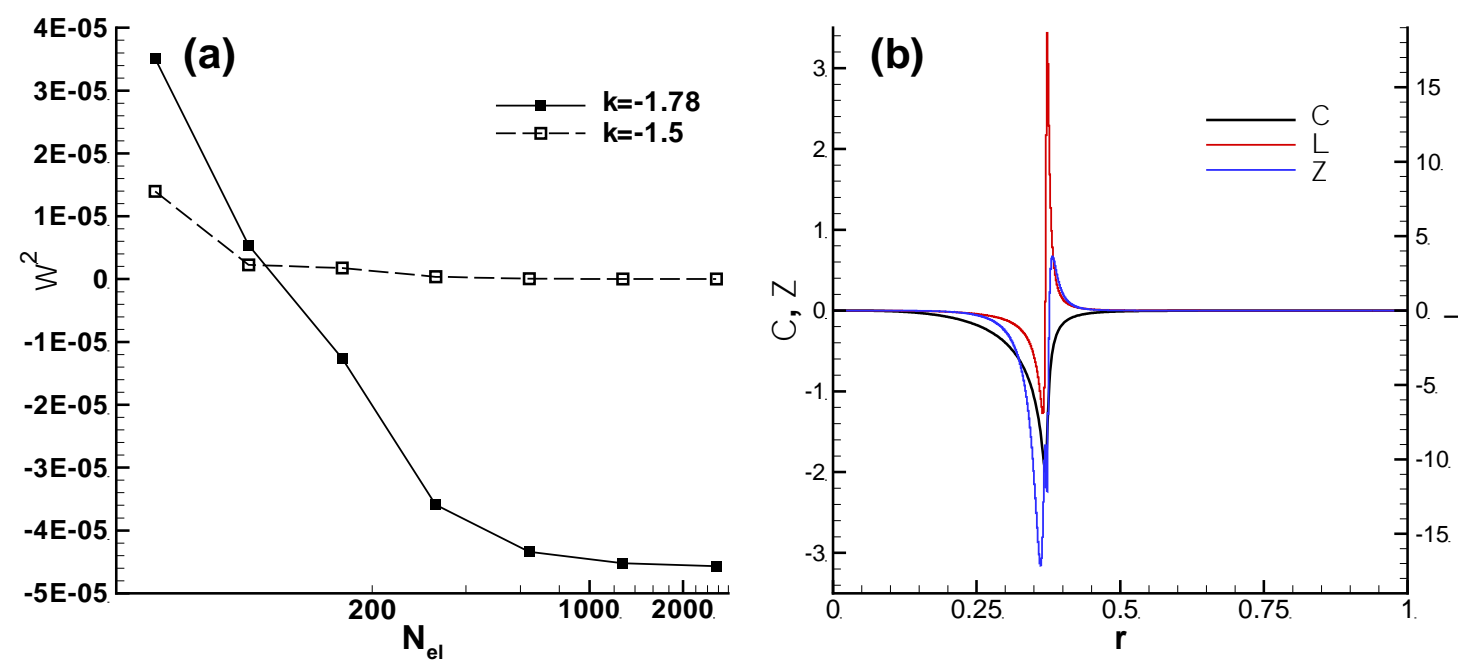

Figure 2. Results on the smallest- 2 eigenvalues computed with the $\mathrm{X}, \Lambda, \mathrm{Z}$-system applied to the two $m=4$ harmonics of the inhomogeneous cylindrical equilibrium. The scan of radial resolution is shown in (a), and the eigenfunction for the unstable case computed with 640 elements is shown in (b). The results indicate real frequencies for all $\mathrm{k}=1.5$ computations and for the $N_{e l}=40$ and 80 computations at $\mathrm{k}=1.784$ and positive growth rates for the $N_{e l} 160$ computations at $\mathrm{k}=1.784$. 

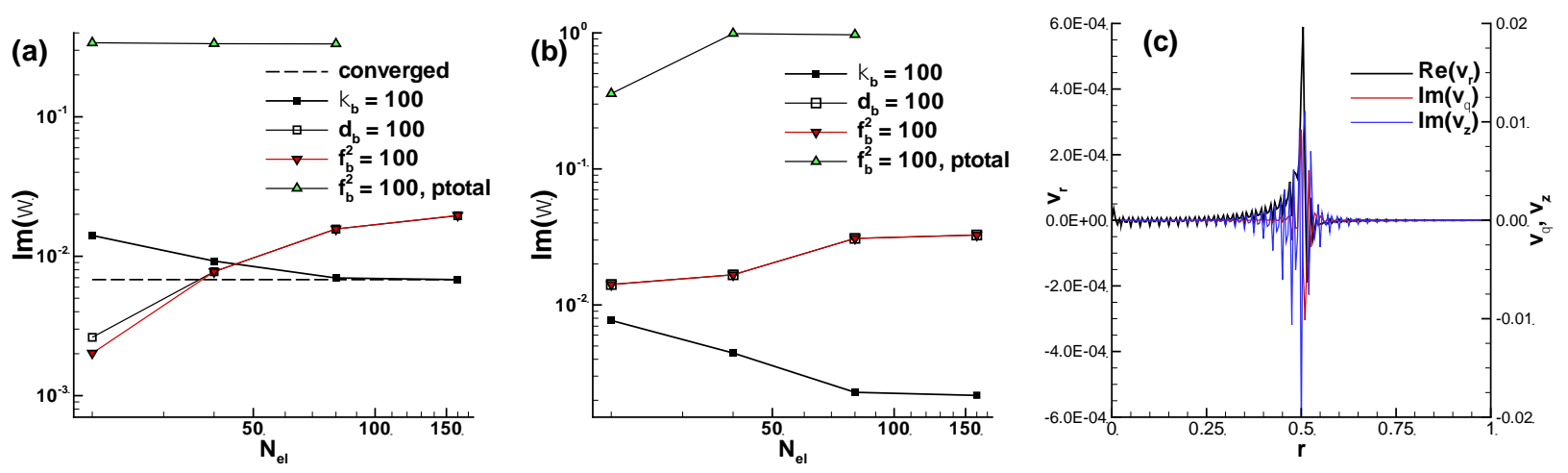

Figure 3. Eigenmode results computed with the first-order $\mathbf{v}, \mathbf{b}, p$-system for $m=4$ and (a) $\mathrm{k}=1.784$ and (b) $\mathrm{k}=1.5$. The growth rate is plotted for the fastest growing mode from each computation. All computations use quartic expansions for $\mathbf{v}$ and $\mathbf{b}$. Computations that use the $\nabla \nabla \cdot \mathbf{b}$ divergence control are labeled with $\quad b$. Those with the discontinuous cubic auxiliary scalar $\phi$ are labeled with $d_{b}$ (parabolic control) and with $f_{b}^{2}$ (hyperbolic control). Computations using the discontinuous, cubic total pressure are labeled as ptotal. Components of the fastestgrowing $\mathrm{k}=1.5$ eigenmode for $N_{e l}=40$ and the $\nabla \nabla \cdot \mathbf{b}$ divergence control are shown in (c). 


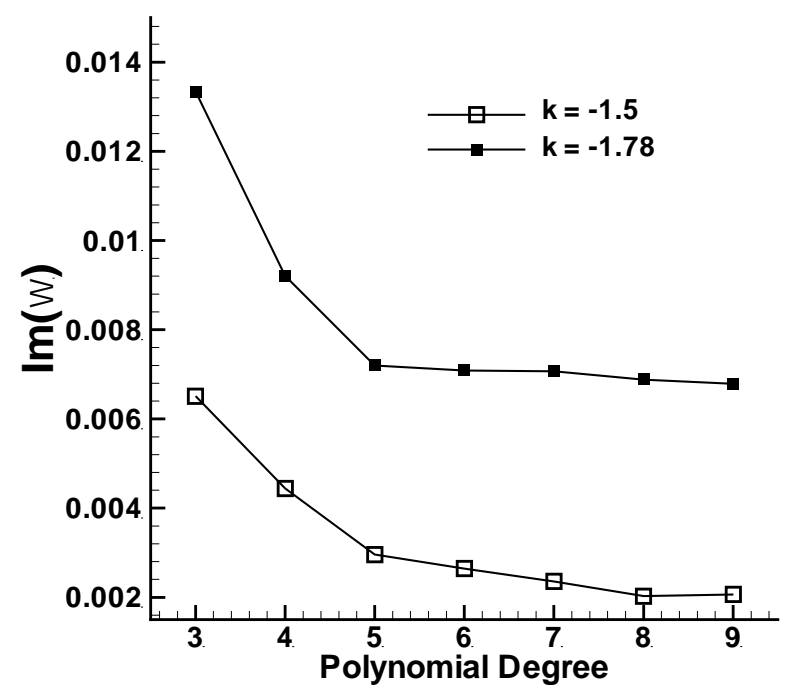

Figure 4. Computed eigenvalue results for the two $\mathrm{m}=4$ harmonics with $N_{e l}=40$ and the $\nabla \nabla \cdot \mathbf{b}$ divergence control, varying the degree of the continuous polynomial bases for the $\mathbf{v}, \mathbf{b}$, and $p$ fields. 

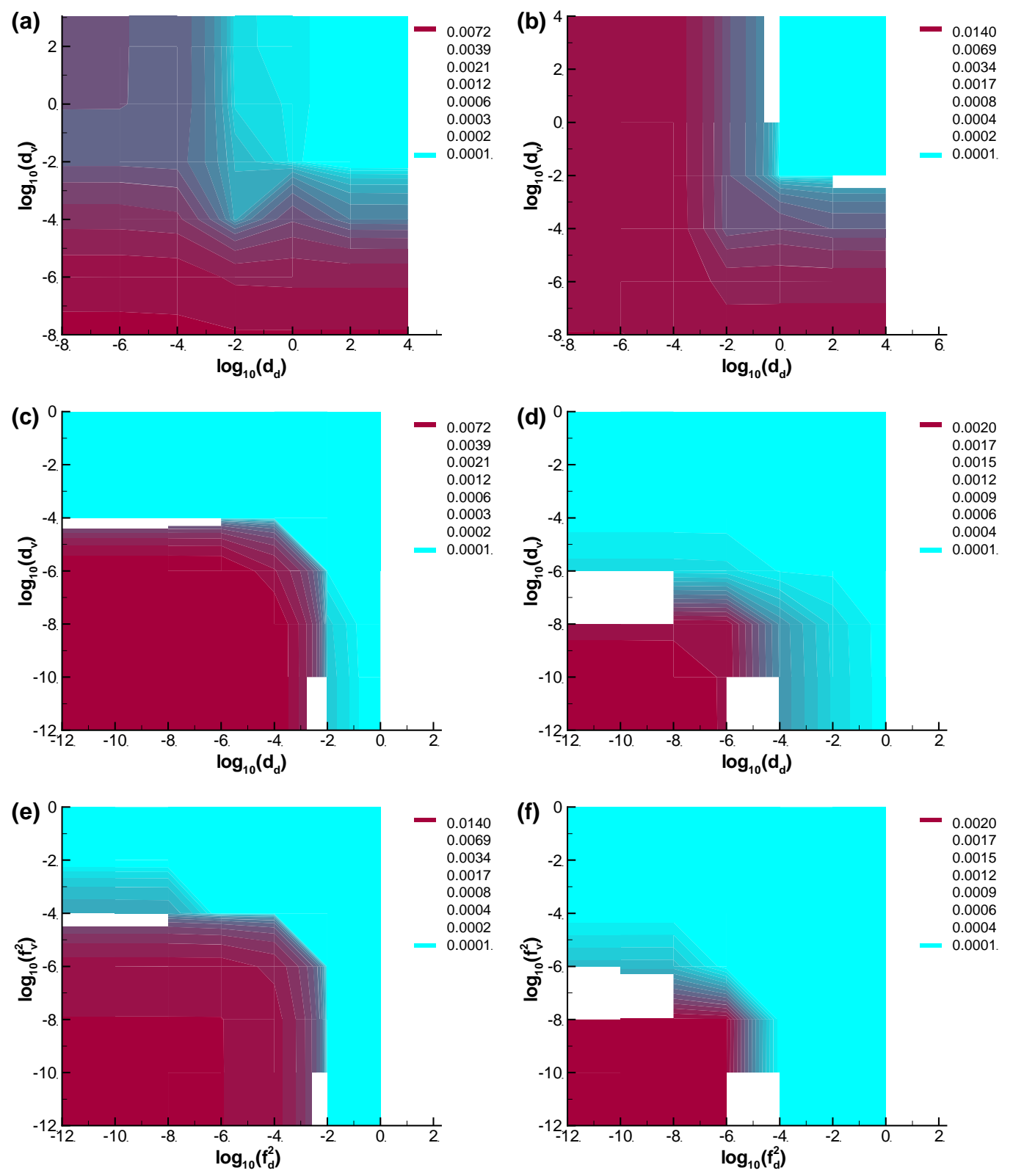

Figure 5. Results on the largest growth rate from $\mathrm{m}=4, N_{e l}=20$, eigenmode computations for the first-order $\mathbf{v}, \mathbf{b}, p$-system with auxiliary projections and responses for flow divergence and parallel vorticity. All computations have $\mathbf{v}, \mathbf{b}$, and $p$ expanded as continuous quartic polynomials, and $\sigma$ and $\lambda$ have just the quartic Chebyshev polynomial in each element. Frames (a-b) have parabolic projections with the $\nabla \nabla \cdot \mathbf{b}$ divergence control; $\mathrm{k}=1.5$ in (a), and $k=1.784$ in (b). Frames (c-d) have parabolic projections with the parabolic $\nabla \cdot \mathbf{b}$ control using $\phi$ expanded in discontinuous cubics; $\mathrm{k}=1.5$ in (c), and $\mathrm{k}=1.784$ in (d). Frames (e-f) have hyperbolic projections with the hyperbolic $\nabla \cdot \mathbf{b}$ control using $\phi$ expanded in discontinuous cubics; $\mathrm{k}=1.5$ in (e), and $\mathrm{k}=1.784$ in (f). 

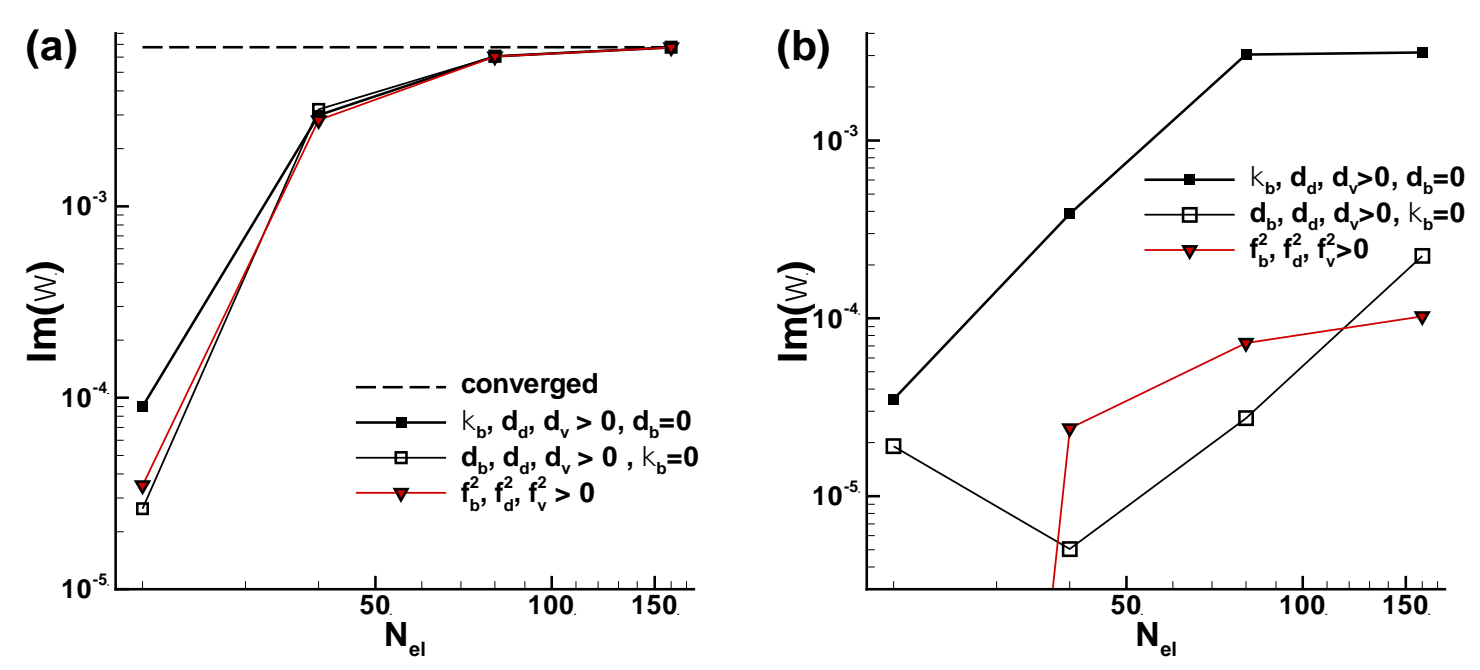

Figure 6. Eigenmode results for the fastest growing $\mathrm{m}=4$, (a) $\mathrm{k}=1.784$ and (b) $\mathrm{k}=1.5$ modes computed with quartic $\mathbf{v}, \mathbf{b}$, and $p$ expansions and with the auxiliary flow-divergence and parallel-vorticity projections. Traces with solid black squares use parabolic projections and the $\nabla \nabla \cdot \mathbf{b}$ divergence control, traces with open squares use parabolic projections and cubic $\phi$, and red traces with gradient symbols use hyperbolic projections and cubic $\phi$. 


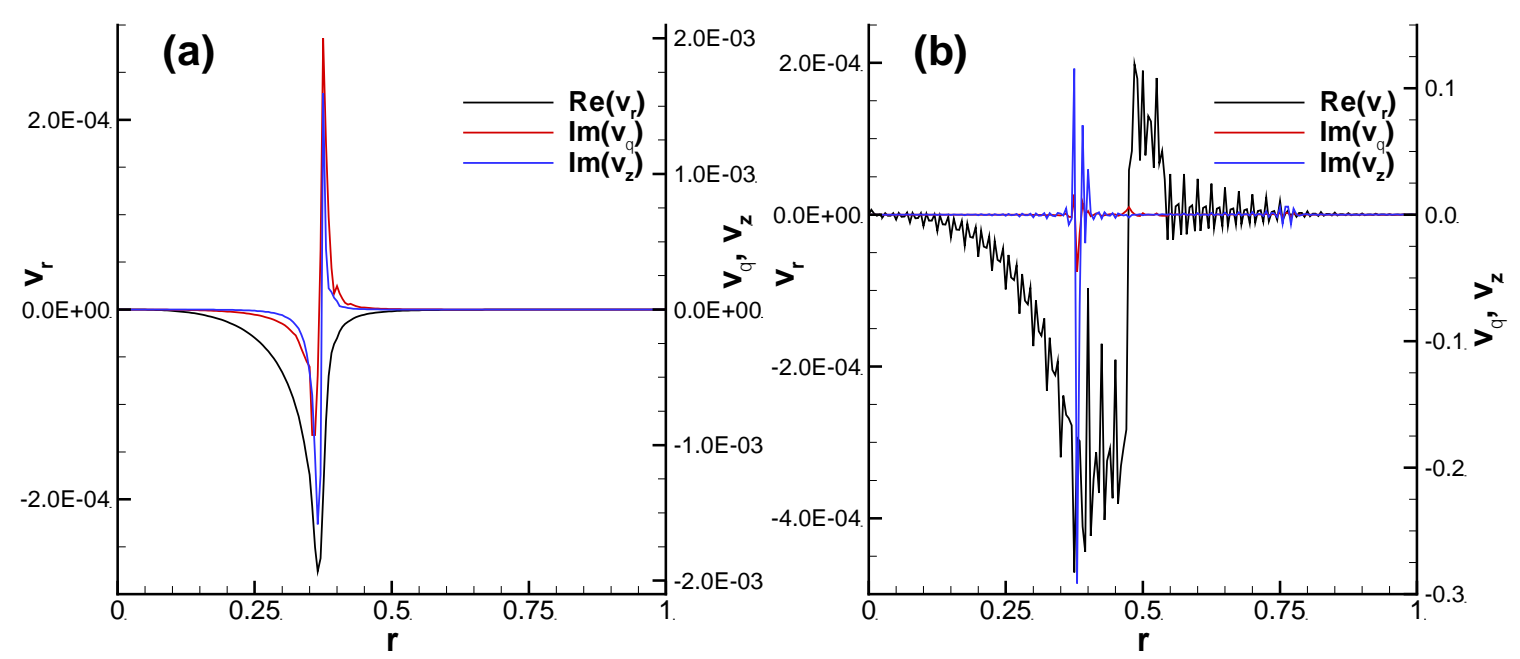

Figure 7. Comparison of growing $\mathrm{m}=4$ eigenfunctions for (a) $\mathrm{k}=1.784$ and (b) $\mathrm{k}=1.5$ from eigenmode computations with $N_{e l}=40$, quartic polynomials for $\mathbf{v}, \mathbf{b}$, and $p$, and hyperbolic auxiliary responses. 


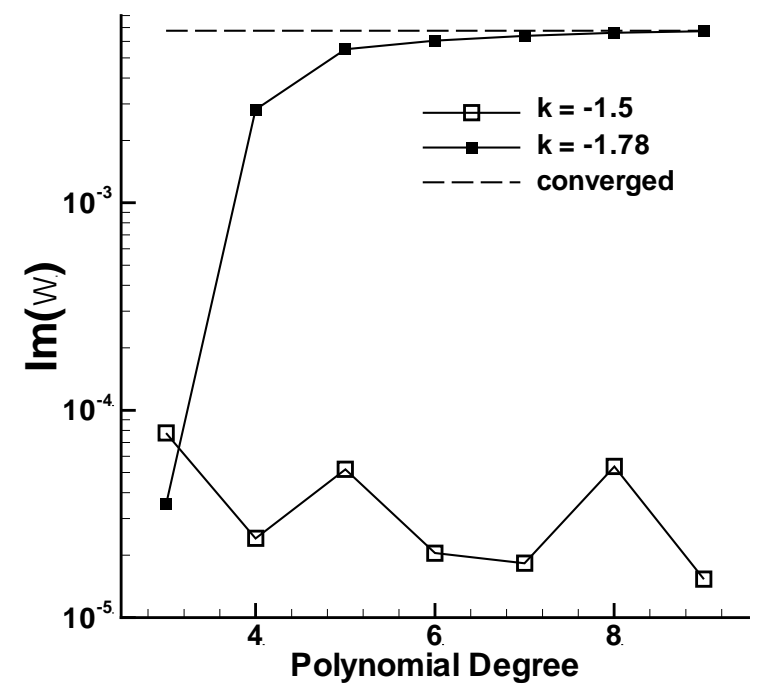

Figure 8. Computed eigenvalue results for the two $\mathrm{m}=4$ harmonics with $N_{e l}=40$ and the hyperbolic flow-divergence and parallel-vorticity projections. The horizontal axis shows the degree of the continuous expansions for $\mathbf{v}, \mathbf{b}$, and $p$. It is also the degree of the single Chebyshev polynomial used for the auxiliary projections. 

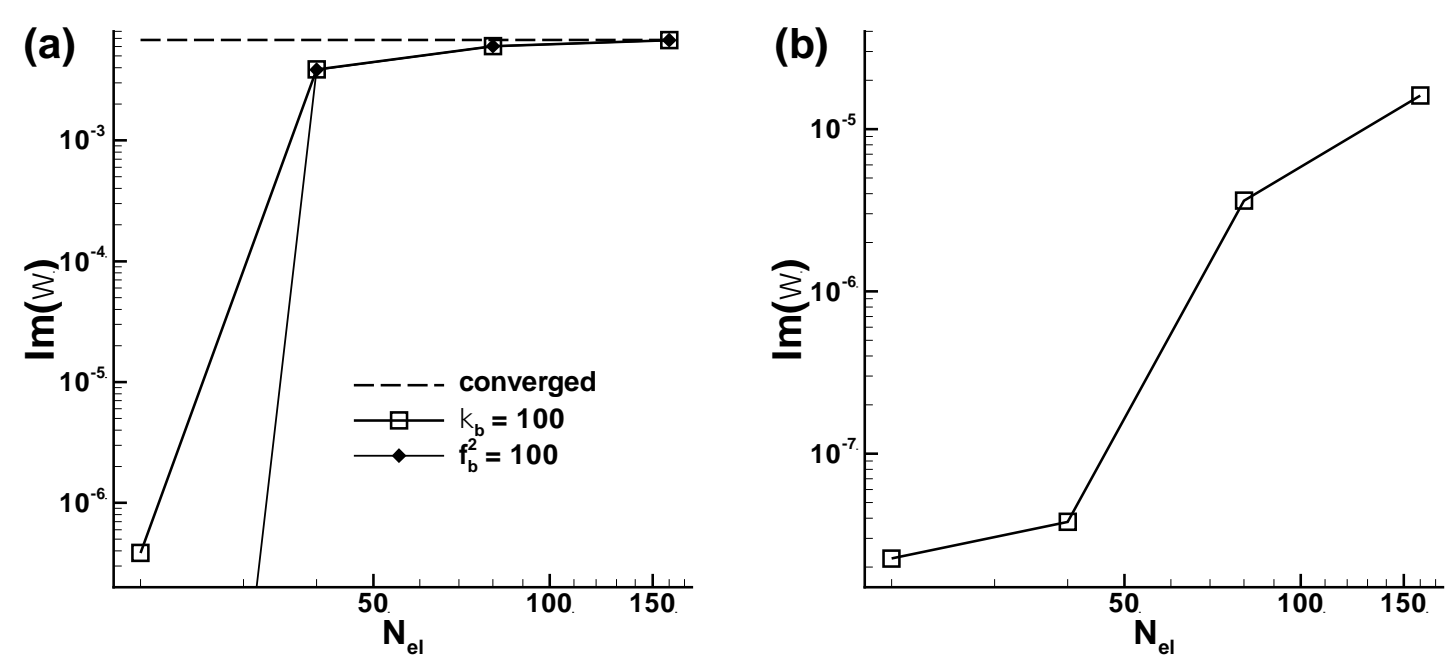

Figure 9. Growth rates from eigenmode computations for $m=4$, (a) $k=1.784$ and (b) $\mathrm{k}=1.5$ with continuous cubic expansions for $\mathbf{v}$ and continuous quartic expansions for $\mathbf{b}$ and $p$. Traces with open squares use the $\nabla \nabla \cdot \mathbf{b}$ divergence control, and the trace with solid diamonds uses the auxiliary $\phi$ with hyperbolic response. Computations for $\mathrm{k}=1.5$ with the hyperbolic auxiliary $\phi$ produce only stable modes. 
(a)

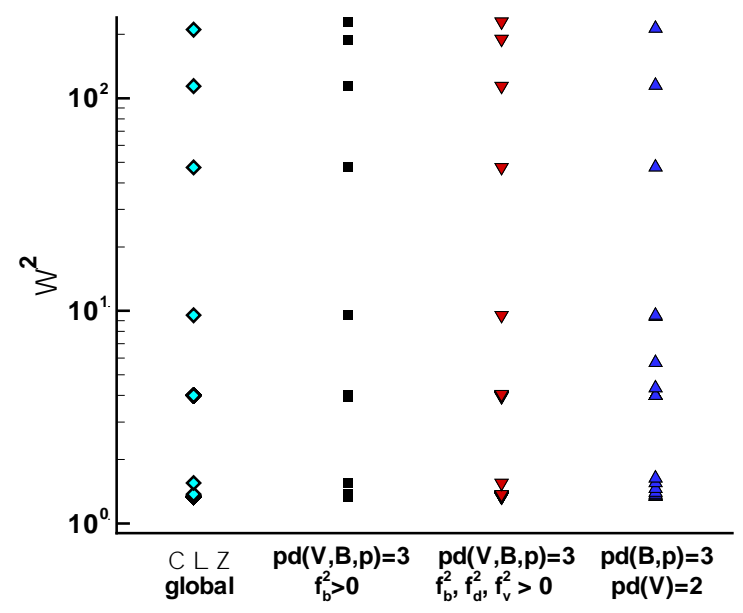

(b)

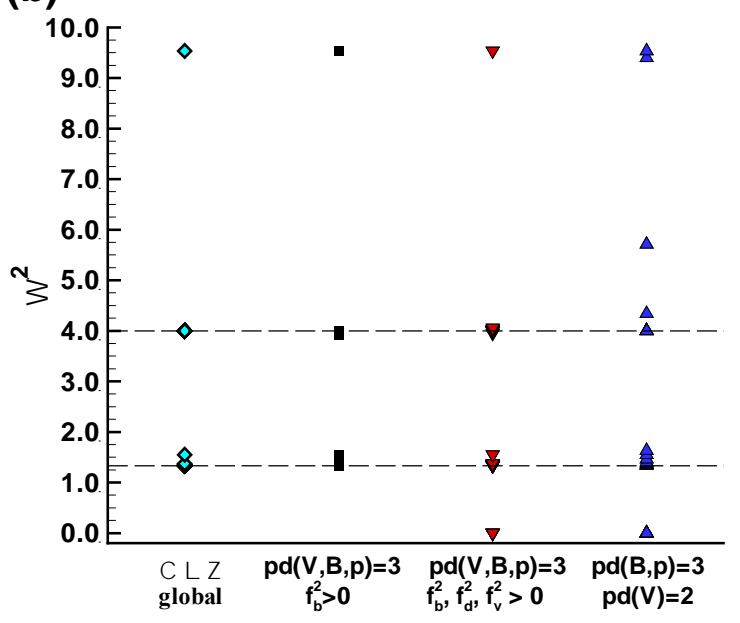

Figure 10. Comparison of wave spectra from eigenmode computations with the uniform cylindrical equilibrium. Frame (b) expands the region in (a) near the sound accumulation point and the Alfvén spectrum (dashed lines). The four computations use global Chebyshev polynomials for the X, $\Lambda$, Z-system (cyan diamonds), continuous cubic elements for the $\mathbf{v}, \mathbf{b}, p$ system without flow-divergence and parallel-vorticity projections (black squares), with projections (red gradients), and with quadratic v-expansion without projections (blue triangles). 


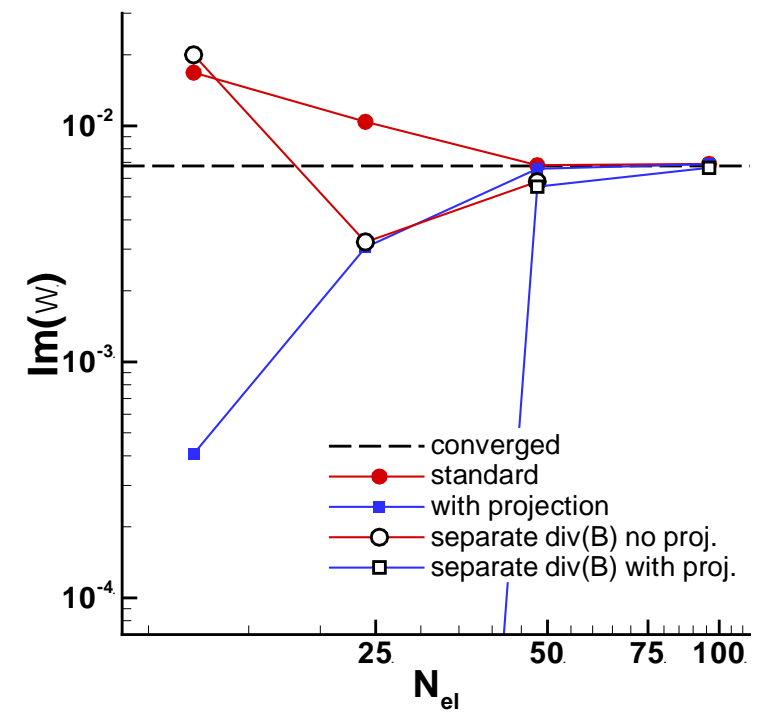

Figure 11 . Growth rates for the $\mathrm{m}=4, \mathrm{k}=1.784$ cylindrical interchange mode from timedependent computations with biquartic expansions for the physical fields. The horizontal axis is the number of elements in the radial direction, and in each computation, there are half as many elements in the azimuthal direction. Traces with circular symbols are results without the projections for flow divergence and parallel vorticity, and those with square symbols have these projections. 

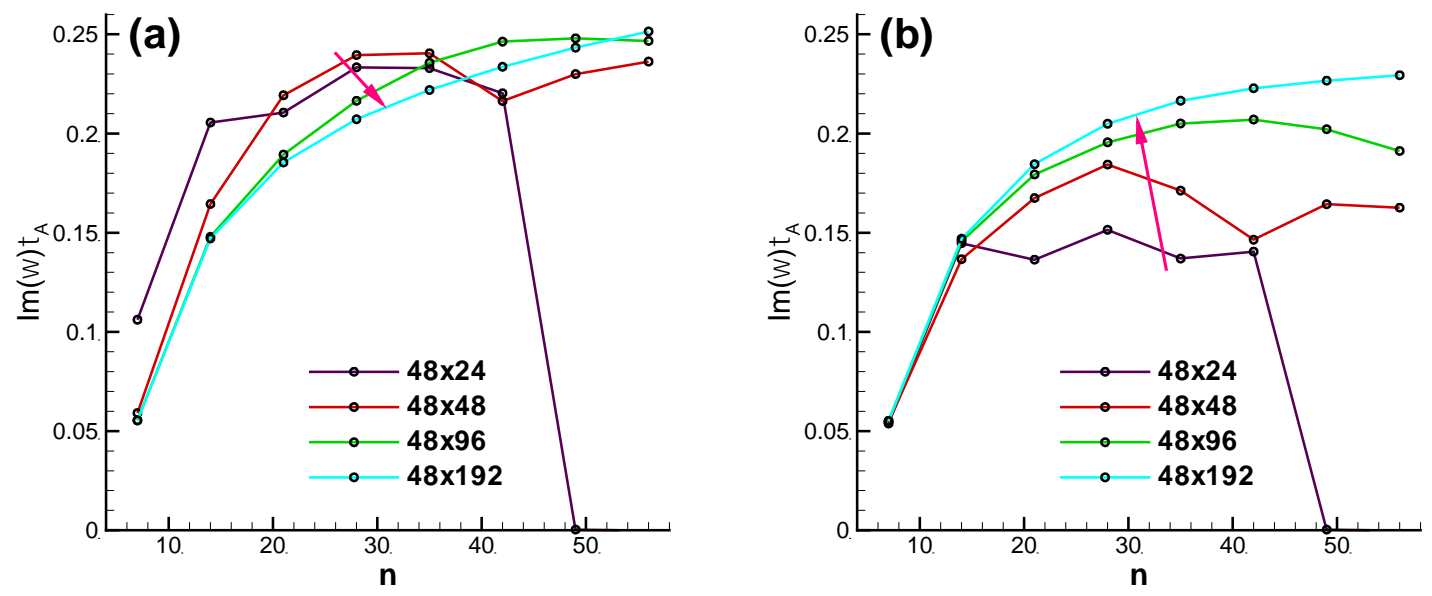

Figure 12. Results of time-dependent linear ballooning-mode computations. The spectra in (a) are produced without the flow-divergence and parallel-vorticity projections, and those in (b) incorporate the projections. Labels indicate the mesh resolution (flux-normal $\times$ azimuthal) for each computation, and the arrows show the trends with increasing resolution. 

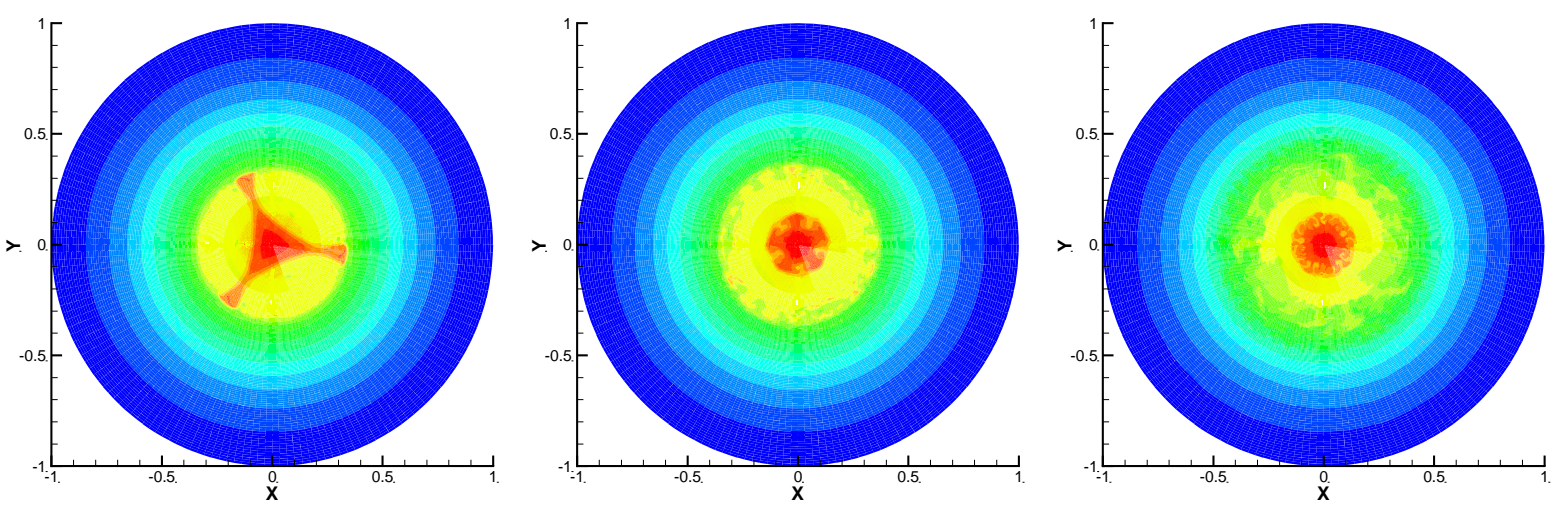

Figure 13. Contours of pressure from the nonlinear computation with flow-divergence and parallel-vorticity projection. The three frames are from $t=4800$ (left), $t=5600$ (center), and $t=7250$ (right). 
(a)

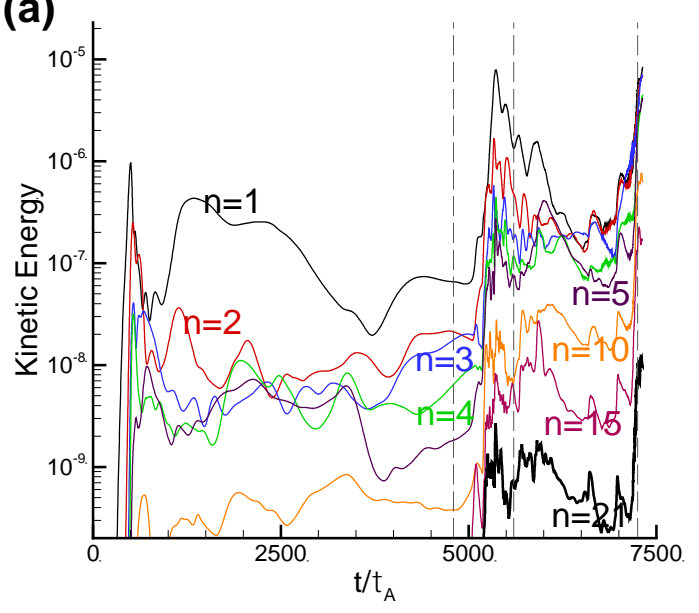

(b)

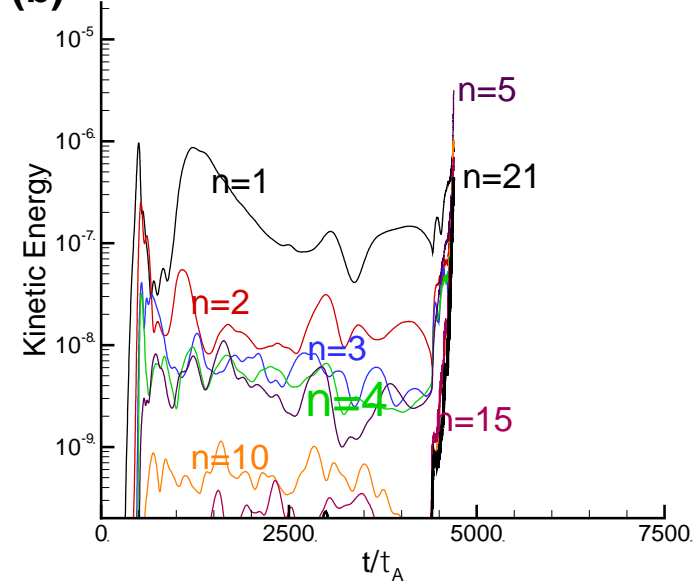

Figure 14. Evolution of kinetic energy spectra from nonlinear computations (a) with flowdivergence and parallel-vorticity projections and (b) without the projections. The vertical lines in (a) indicate the time slices at which pressure is plotted in Fig. 13. 